\title{
Cortical inhibitory network selects cerebellar signals for movement initiation
}

\author{
Abdulraheem Nashef ${ }^{1}$, Oren Cohen ${ }^{1}$, Steve I. Perlmutter ${ }^{2}$ and Yifat Prut $^{1,3^{*}}$ \\ ${ }^{1}$ Department of Medical Neurobiology, IMRIC and ELSC, The Hebrew University, Hadassah Medical \\ School, Jerusalem 9112102, Israel \\ ${ }^{2}$ Department of Physiology \& Biophysics and the Washington National Primate Research Center, Box \\ 357330, University of Washington, Seattle, Washington 98195, USA \\ ${ }^{3}$ Lead contact \\ *Correspondence: yifatpr@ekmd.huji.ac.il
}

Running title: inhibitory control of motor cortical activity

Number of figures: 6

Number of Supplemental figures: 4 
2 The onset of voluntary movements is driven by coordinated firing across a large

3 population of motor cortical neurons. This pattern of activity is determined by both

4 local interactions and long-range corticocortical and subcortical inputs. The way

5 remote areas of the brain communicate to effectively drive movement is still unclear.

6 We addressed this question by studying an important pathway through which the cerebellum communicates, via the motor thalamus, with the motor cortex. We found that similar to the sensory cortices, thalamic input to the motor cortex triggers feedforward inhibition by directly contacting inhibitory cells via particularly effective GluR2- lacking AMPA receptors blocked by NASPM. Based on these results, we constructed a classifier for SCP-responsive cortical cells to identify pyramidal and PV interneurons and study their role in controlling movements. The findings indicate that PV and pyramidal cells are co-driven by TC input in response to activation of the CTC pathway. During task performance, PV and pyramidal cells had comparable relations to movement parameters (directional tuning and movement duration). However, PV interneurons exhibited stronger movement-related activity that preceded the firing of pyramidal cells. This seemingly counterintuitive sequence of events where inhibitory cells are recruited more strongly and before excitatory cells may in fact enhance the signal-to-noise ratio of cerebellar signals by suppressing other inputs and prioritizing the excitatory synchronized volley from the TC system which occurs at the right time to overcome the inhibitory signal. In this manner, the CTC system can shape cortical activity in a way that exceeds its sheer synaptic efficacy.

\section{KEYWORDS}

Thalamocortical; Motor control; Feed-forward inhibition; lontophoresis; PV+ interneurons; Cerebellum; Non-human primates

\section{INTRODUCTION}

Motor commands for voluntary movements result from the integration of information from multiple cortical and subcortical sources by the motor cortex (Middleton and 
Strick, 2000). Numerous studies have explored the role of local motor cortical dynamics in producing actions (Churchland et al., 2012; Kaufman et al., 2014; Kaufman et al., 2013; Shenoy et al., 2013). However, the way remote brain regions communicate with the motor cortex and take an effective part in driving movements is still unclear. To investigate this question, we focused on the cerebellarthalamocortical (CTC) pathway, through which the cerebellum influences motor cortical activity. Cerebellar signals participate in shaping motor cortical commands and play a specific role in the regulation of motor timing coordination (Bastian et al., 1996; Holmes, 1917, 1939; Ivry and Keele, 1989). This means that despite the large distance cerebellar information needs to travel, and the limited number of synapses onto motor cortical neurons (Bopp et al., 2017; Schoonover et al., 2014) this pathway exerts online impact on motor output. In sensory (Cruikshank et al., 2007; Gabernet et al., 2005) and frontal (Delevich et al., 2015) cortical areas, thalamocortical inputs produce feedforward inhibition (FFI) of cortical activity, which dictates a temporal window for information processing (Pouille and Scanziani, 2001). In the motor system, there is indirect evidence for a cerebellar role in braking and inhibiting motor cortical circuitry (Hore and Flament, 1988; Nashef et al., 2018a) and unwarranted movements (Babinski, 1913). We hypothesized that a feedforward mechanism in the CTC system may mediate and prioritize the role of cerebellar signals in timing the onset of voluntary movements.

To test this hypothesis, we combined stimulation of the CTC pathway, electrophysiological recordings of motor cortical neurons, and pharmacological perturbations of transmission through this pathway in behaving primates. This enabled us to positively identify GABAergic interneurons and pyramidal neurons that integrate CTC information in the motor cortex. Based on studies in the sensory system of rodents, where the circuitry of the FFI relies on particularly powerful synapses by TC fibers made selectively onto fast-spiking parvalbumin positive (PV) neurons (Bruno, 2011; Cruikshank et al., 2007; Ji et al., 2015; Yu et al., 2019), we suggest that these interneurons are in fact PV cells. The properties of this subset of cells were used to construct a classifier for PV and pyramidal neurons out of all the cells responsive to stimulation of the superior cerebellar peduncle (SCP). During task performance, the movement-related activity of PV neurons preceded that of pyramidal cells and their directional tuning was slightly broader. In addition, the 
activity of PV cells was strongly correlated with the kinematic properties of the movements. Finally, the firing of PV and pyramidal cells was specifically correlated around movement onset, indicating strong and specific cross-interactions between these cell groups.

Our results suggest that the CTC system relies on FFI in the motor cortex in a way similar to the circuitry found in cortical sensory areas of rodents. The functional consequences of this circuitry are the strong coupling between SCP-responsive PV and pyramidal cells and the task-related activity of PV cells preceding that of the pyramidal cells. We posit that this counterintuitive sequence of events serves to assign a high priority to cerebellar signals over other competing inputs. In this manner PV neurons play an important role in steering cortical activity at movement onset to efficiently translate cerebellar input into timing signals for motor commands. Finally, the inhibitory control exerted by the cerebellum on motor output maybe mediated, in part, by the specific potent recruitment of motor cortical interneurons.

\section{RESULTS}

Our previous findings indicated that cerebellar signals routed through the CTC system efficiently recruit an extensive population of cortical cells throughout the motor cortex (Nashef et al., 2018a). Here we aimed to dissect the local cortical circuitry that integrates these signals to elucidate the involvement of different subclasses of cortical neurons in controlling the timing of movements. To do so, we took advantage of the organizational principles of the TC system in the sensory and frontal areas of rodents where TC axons make distinctive connections with excitatory and inhibitory cells (Cruikshank et al., 2007; Gabernet et al., 2005). We implanted stimulating electrodes in the SCP (Figure 1 ) of monkeys $(n=3)$ that were trained to wear an exoskeleton (Kinarm system) and perform a center-out reaching task (Figure 1A-B). The behavioral task used here was designed to encourage the monkeys to move as fast as possible by predicting the time of the "go" signal (Nashef et al., 2019). Neural activity was recorded from arm-related motor cortical areas using multiple single electrodes in 3 monkeys (Figure 1C); in two of these animals, neurons were also tested using a 3-barrel recording pipette (Thiele et al., 2006). This experimental approach (Figure 1D) enabled us to record the activity of 
motor cortical cells during movement, identify their response to CTC activation, and in some cases pharmacologically perturb their activity by iontophoretic application of neuroactive compounds.

\section{Identifying motor cortical neurons that integrate cerebellar signals}

The connectivity pattern between TC fibers and specific classes of neurons has a major impact on the way subcortical input affects cortical activity. In sensory areas, TC inputs contact both pyramidal and PV cells, thus generating a feedforward inhibitory connection. In fact, the contacts between TC axons and cortical inhibitory interneurons are almost exclusively confined to PV cells (Ji et al., 2015), and these synapses are mediated by a specific type of AMPA receptor which lacks the GluR2 subunit (Hull et al., 2009). These receptors are unique in their calcium permeability, their particularly large synaptic currents, and the specificity of the blocker 1naphythyl acetyl spermine or NASPM (Hull et al., 2009). Importantly, GluR2-lacking AMPA receptors are not found on pyramidal cells of the adult brain (Geiger et al., 1995; Kumar et al., 2002; McBain and Dingledine, 1993).

Based on these data we employed a two-step identification criterion for PV neurons in the CTC pathway (Figure 2A). We identified cells $(n=360)$ that were part of the CTC system by their early excitatory response to single pulse SCP stimulation (Nashef et al., 2018a). Out of this group, we tested 58 neurons for changes in the SCP-triggered response after blocking GluR-2 receptors by iontophoretically applying NASPM from the barrel of the recording pipette. Cells that decreased their early excitatory response to SCP stimulation in the presence of NASPM were identified as PV interneurons whereas cells that either maintained or increased their evoked response were defined as pyramidal cells (Figure 2B). Of the 58 tested cells, $28 \%(n=16)$ decreased their response in the presence of NASPM, and were classified as PV interneurons. The remaining 42 cells (72\%) maintained (or increased) their response to stimulation, and were classified as pyramidal cells (Pyr). In the presence of NASPM, the SCP-triggered response of PV interneurons exhibited a significant decrease in response amplitude (Figure $2 \mathrm{C}$ ), pre-stimulus firing rate, area under the excitatory peak of the response curve, and area of the inhibitory phase of the response (Wilcoxon's signed-rank test; $p<0.001$ for all parameters). In contrast to these robust changes, the response pattern of pyramidal neurons to SCP stimulation was unaffected (Figure $2 \mathrm{C}$ ). In addition, neurons 
classified as PV cells had significantly narrower action potential (AP) widths (measured as the time from trough to peak) than pyramidal neurons (Pyr $=0.46 \pm 0.02$ $\mathrm{ms} ; \mathrm{PV}=0.35 \pm 0.03 \mathrm{~ms}$; Wilcoxon's rank sum test, $p<0.005)$. These findings thus indicate that in the CTC system of primates, TC fibers connect directly to both excitatory (pyramidal) and inhibitory (presumably PV) cells in a scheme comparable to the FFI reported for sensory systems in rodents.

In the second step of the identification process, the responses of the cortical cells to SCP stimulation, their background firing rate and AP width were used to construct a mixed classifier (naïve Bayesian and support-vector machine-SVM (Cortes and Vapnik, 1995)) which was applied to the remaining dataset of 302 SCPresponsive cells (Figure 2D). The most clear-cut feature for classification (beyond the actual response to stimulation, which was set as an initial condition) was the width of the waveform, which alone achieved $70 \%$ classification accuracy. The prestimulus firing level and the magnitude of excitation triggered by SCP stimulation (area under the response curve) improved the classification accuracy to $76 \%$ (Pyr: $76.2 \% ; \mathrm{PV}: 75 \%)$. Although the pre-stimulus firing level and the magnitude of excitation were not significantly different for the training population between the two classes, the three features used here showed a significant multivariate relationship with cell class (multivariate analysis of variance, $p<0.005$; degrees of freedom=57). Overall, we classified 199/302 (66\%) SCP-responsive cortical cells as pyramidal cells and the remaining 103 (34\%) as PV cells (Figure 2E). For each neuron, we calculated the ratio of the distance between the neuron's location in the clustering space (Figure 2E) to the center of the other cluster over the distance to the neuron's allocated cluster center. We then averaged the ratios for all neurons to define a clustering score as a measure of the goodness of clustering. This score was compared to the distribution of scores obtained in a similar manner on datasets where cluster allocation was random, while keeping the counts for each cell class fixed. The actual score (computed for the real dataset) was located far from the distribution of values calculated for shuffled datasets (Figure $2 F, p<<0.001$ ), supporting the classification approach used here. In another test, we compared the results of classification from the training set to a "blind" classification procedure using a Gaussian mixture distribution classifier of two subpopulations. We found that the clustering based on the training dataset yielded higher clustering scores than the 
blind classification ( $p<0.02$; Figure 2G). Thus, the training-based classifier used here outperformed untrained clustering methods used in the past (Katai et al., 2010; Trainito et al., 2019).

\section{Movement-related activity of PV interneurons precedes activity of pyramidal} cells

We compared the movement-related activity of identified cells during task performance. In our dataset, inhibitory cells had a stronger and more reliable response to SCP stimulation (Figure S1). This is consistent with previous studies showing that the cortical response to activation of the CTC system is dominated by inhibition (Hore and Flament, 1988; Nashef et al., 2018a). This differential recruitment of specific cell types by CTC signals was expected to promote distinct patterns of activity in these cells during movement. To test this hypothesis, we compared the response pattern of pairs of PV and Pyr cells recorded simultaneously on one or two electrodes. Simultaneous recordings eliminate the possibility of confounds due to session-to-session variability in motor behavior. We found significantly different movement-related properties in PV and Pyr neurons (Figure 3). PV cells had a higher background firing rate (Figure 3A, Wilcoxon's signed-rank test; $\mathrm{p}<0.05)$, and a higher task-related response amplitude around movement onset time (Figure 3B; $p<0.003$ ). However, the most remarkable difference between the two cell types was the tendency of PV cells to increase their movement-related firing earlier than Pyr cells. Figure 3C shows an example of movement-related activity of a PV cell and a Pyr cell recorded simultaneously. In this example, the onset of the increased movement-related activity of the PV cell preceded that of the Pyr cell by $360 \mathrm{~ms}$ and the peak firing was $4.7 \mathrm{sp} / \mathrm{s}$ higher. The propensity of PV cells for an earlier and larger amplitude response was also present at the population level (Figure 3D). To compensate for the higher activity of PV compared to Pyr cells, we normalized the firing rate of each neuron to its peak activity (which was thus defined as $100 \%$ ) and identified the time at which activity reached $20 \%$ of the maximal peak value (Figure 3E). We found that PV neurons reached this threshold about $200 \mathrm{~ms}$ before pyramidal cells.

\section{Encoding of motor parameters by different cell classes}


Next, we tested whether the correlation between movement-related signals and specific kinematic parameters was different for the two cell classes. We found that the tuning of PV and Pyr cells for the direction of arm movement had similar widths $(p<0.23)$. The same was true for other measures for tuning selectivity, such as the half-width at half height $(\mathrm{HWHH})$ and the orientation selectivity (measured as the 1circular variance) of the tuning curves, neither of which was significantly different for PV and Pyr cells ( $p<0.63$ and $p<0.28$ respectively). In addition, we found no differences in the distribution of PDs between the two cells types (two-sample test for circular data, $p=0.61)$ or in the tendency for tuning $\left(X^{2}\right.$ test; $p=0.09 ; X^{2}=2.17$; degrees of freedom=1).

Next, we tested for correlations between the activity of individual cells and the kinematic parameters of the movements performed. Figure 4A shows the correlation throughout a trial of the activity of a PV and a pyramidal cell with movement duration (MD: the time between movement onset and when the cursor entered the peripheral target). Long before movement started, the activity of the PV (but not the pyramidal) cell was negatively correlated, on a trial-to-trial basis, with the time of the subsequent movement (Figure 4B; note that the positive correlation after movement is trivial, since this time window overlaps with the time of movement). This propensity was also present at the population level; PV cells had a stronger and earlier correlation with MD compared to the Pyr cells (Figure 4C). This was evident both at the average level of correlation and the fraction of the significant cells out of the entire population (Figure S2). Since PV and Pyr cells differed in their rate levels we tested whether cells with enhanced task-related rate modulation also expressed a stronger rate-toMD correlation (Figure 4D). The two parameters were significantly related for PV cells (Pearson $n r=-0.3 ; p<0.003$ ) whereas the correlations expressed by pyramidal cells was independent of rate modulations (Pearson $n r=-0.11 ; p<0.18$ ).

Finally, we investigated the relationship between single cell activity and movement velocity by using the instantaneous hand velocity and acceleration to build a linear model to account for the trial-to-trial firing of the recorded cells (Medina and Lisberger, 2007). Velocity made a significantly higher contribution to predicting PV firing than Pyr firing (Figure 4E). In addition, the firing-to-behavior time lag required for the best model fit for $\mathrm{PV}$ cells was more negative than for the Pyr cells 
(Figure 4F), consistent with our previous finding that PV task-related firing preceded that of Pyr cells.

Taken together, these results suggest that the movement-related activity of PV cells starts before that of excitatory cells, but nevertheless robustly encodes motor parameters such as direction, movement duration and velocity. This finding assigns a dominant role to PV neurons in coordinating not only the activity of pyramidal neurons, but also in steering motor behavior.

Synchronous activation of PV and Pyr cells

SCP-responsive PV and Pyr cells are not independent channels but rather embedded within a rich cortical network which makes them likely to interact. These interactions can amplify or suppress incoming cerebellar signals in a task-dependent manner. We tested this possibility using two different approaches. First, we estimated the correlated firing (spike-count correlation) of simultaneously recorded PV and Pyr cells triggered by SCP stimulation. This analysis was only carried out on cells recorded simultaneously from two different electrodes, to prevent any masking of synchrony due to super-positioning of action potentials (Bar-Gad et al., 2001). We found that SCP stimulation triggered cell-specific synchronization patterns. Pairs of PV and pyramidal cells expressed an early increase in correlated firing followed by a decrease in co-firing which occurred at time lags consistent with PV-to-Pyr inhibition (Figure 5A). This correlation pattern is consistent with the expected correlation calculated for a simulated TC circuit with FFI (Figure 5B-C and Figure S3) constructed based on previously published parameters (Cruikshank et al., 2007; Izhikevich, 2003; Kremkow et al., 2010). By contrast, the synchronizing effect of SCP stimulation on pairs of pyramidal cells was substantially weaker (Figure 5D). Here again, the results were consistent with the correlation calculated for the simulated Pyr-Pyr pairs using similar conditions to our stimulation protocol in terms of number of sweeps (Figures 5E-F).

Next, we searched for traces of this SCP-triggered correlated firing in the activity of the cells during task performance. During natural behavior the activation of the CTC pathway is sparse and dispersed compared to the tightly synchronized activation in response to SCP stimulation. Nonetheless, we found that around movement onset, cells that are part of the CTC system were co-driven in a cell- 
specific manner consistent with their activation pattern in response to SCP stimulation. Specifically, pyramidal and PV cells expressed time-varying synchrony locked to movement onset (Figure 6A), whereas pairs of Pyr cells were generally and consistently uncorrelated (Figure 6B). The correlation pattern between PV and Pyr cells consisted of a positive correlation before onset and after the offset of movement and a decorrelation during movement (Figure $6 \mathrm{C} ; \mathrm{p}<0.02$ ), whereas the correlation of Pyr cells was not significantly modulated between these epochs (Figure $6 C ; p=0.72$ ). The observed correlation for pairs of PV/PV and Pyr/PV were similar in pattern to that found in response to evoked CTC activation, whereas the Pyr/Pyr correlation was substantially weaker. Similar results were obtained when measuring the correlated firing only in trials directed towards the preferred direction (Figure S4). Finally, to test the effect of the different rate levels of PV and Pyr cells, we computed the correlated firing between a subset of Pyr-Pyr pairs where at least one of the neurons had a rate level comparable (i.e., equal to or higher than the mean- $1 / 2 S D$ ) to that of the PV cells (Figures $6 D-F$ ). We found that although this subset of pyramidal cells exhibited a similar response amplitude around movement onset as the PV population (Figure 6D), the cells expressed no correlation around movement onset (Figure 6E). In addition, the relationship between the task-related firing of cells and the corresponding level of correlated firing was not significant (Figure 6F). Taken together, these results suggest that in general, the CTC drive is sufficiently strong to induce PV-to-Pyr correlated firing around movement onset, so that the interactions between these cells are further amplified and may thus affect subsequent movement.

\section{DISCUSSION}

283 Motor cortical circuitry integrates multiple inputs and transforms them into descending motor commands. Of these inputs, cerebellar signals are important for motor timing and coordination (Holmes, 1917, 1939; Horne and Butler, 1995). This supposition relies on data obtained from individuals suffering from cerebellar lesions or injury. However, it is unclear how cerebellar outflow, which is funneled through a relatively small anatomical pathway, regulates the timing of motor cortical activity and hence motor actions. This study describes a novel effort to address this question by combining electrophysiological and pharmacological tools to dissect the motor 
cortical circuit that integrates cerebellar signals in behaving primates. We showed that the CTC system follows the same organizational principles as reported for sensory systems; namely, feedforward inhibition through enhanced activation of PV cells compared to pyramidal cells. We further showed that the functional outcome of this arrangement is the earlier onset and tighter relationship to movement duration of the activity of $P V$ cells compared to pyramidal cells. We therefore suggest that the dual, inhibitory-excitatory activation of motor cortical cells by CTC input is important for prioritizing cerebellar signals compared to competing inputs, and that this mechanism enables the CTC system to play a dominant role in timing movement onset. This arrangement assigns a functional role to the potent cerebellar link with the motor cortical inhibitory system.

\section{Combining electrical and pharmacological perturbations to disentangle motor}

cortical circuitry. The lack of information about cell type and its input/output connectivity pattern constitute major obstacles to interpreting the functional consequences of single cell activity recorded in behaving primates. Here we combined two approaches to overcome this limitation. We used SCP stimulation to identify motor cortical cells which integrate cerebellar signals (Nashef et al., 2018a). We then pharmacologically identified PV and pyramidal cells in this population. To do so, we relied on data previously obtained for thalamocortical systems in rodents which showed that thalamic inputs synapse almost exclusively onto pyramidal and PV cells (Hull et al., 2009; Ji et al., 2015) and that these synapses are mediated by GluR2 lacking AMPA receptors on the PV but not the pyramidal cells (Geiger et al., 1995; Jonas et al., 1994; Kondo et al., 1997; McBain and Dingledine, 1993). We classified cells for which the early SCP-triggered response was abolished in the presence of NASPM, an antagonist of GluR2- lacking AMPA receptors, as inhibitory PV interneurons. A possible confound to our interpretation of the NASPM results is whether the TC projection to inhibitory interneurons in the primate motor cortex primarily targets PV cells, as is the case in sensory systems in rodents, since their cortical organization and layer-specific thalamic input are different. The response properties of the interneurons identified here (i.e., higher baseline firing rates, narrow action potentials and monosyantpic connectivity with TC fibers) are all supportive of these cells being PV interneurons. Nonetheless, our functional conclusions are not dependent on the exact subtype of inhibitory cell receiving TC input. 
324 Another possible confound is the non-specific and varied effects of polyamines on

325 NMDA receptors, which depend on the concentration and specific chemical subtype

326 (Rock and Macdonald, 1995). Although NMDA receptors are not an integral part of

327 the FFI circuity (Hull et al., 2009; Kloc and Maffei, 2014), they may play an indirect

328 role in modulating the response pattern of cortical cells to SCP activation, especially

329 the late inhibitory phase. However, NASPM was found to not affect NMDA-mediated

330 activation (Sanderson et al., 2016). Moreover, our analysis of responses to SCP

331 stimulation was restricted to the early excitatory response, which is not affected by

332 NMDA-mediated currents. We therefore conclude that the NASPM-induced

333 attenuation of SCP-triggered responses were primarily mediated by GluR2- lacking

334 AMPA receptors and can thus be used to accurately identify PV cells.

335 We found that AP width reliably distinguished between PV and pyramidal neurons

336 identified by SCP-stimulation and NASPM responses. This criterion is commonly

337 used to distinguish between excitatory and inhibitory cells both in rodents (Bortone et

338 al., 2014; Middleton et al., 2012; Zagha et al., 2015) and in primates (Kaufman et al.,

339 2010; Kaufman et al., 2013; Merchant et al., 2008; Mitchell et al., 2007; Onorato et

340 al., 2019). However, recent findings indicate that pyramidal tract neurons (which are

341 exclusively excitatory cells) can have a narrow AP which is related the existence of

342 Kv3.1b potassium channels (Soares et al., 2017). Our results may not necessarily be

343 at odds with this finding, since our dataset was restricted to SCP-responsive cells;

344 i.e., cells that receive CTC input, and the fraction of PT neurons within this

345 population is unclear (Peters et al., 2017).

Feedforward inhibition (FFI) as a canonical TC mechanism across species and

modalities. We found a large fraction of cells in the motor cortex that received TC input via NASPM-sensitive synapses, indicating inhibitory cells (a ratio of 1-to-2 between SCP-responsive PV to pyramidal cells). The existence of a direct connection between the CTC system and inhibitory interneurons indicates that cerebellar outflow triggers FFI in the motor cortex of behaving primates, similar to what has been observed in sensory and frontal cortical areas of rodents (Cruikshank et al., 2007; Delevich et al., 2015; Gabernet et al., 2005; Swadlow, 2003). The fundamental principle of $\mathrm{FFI}$ is that driving signals simultaneously trigger excitation and inhibition in the target structure. The functional benefits of this arrangement are still not fully understood. Several studies have speculated that it may provide a 
357

358

359

360

361

362

363

364

365

366

367

368

369

370

371

372

373

374

375

376

377

378

379

380

381

382

383

384

385

386

387

388

389

temporally-tight perceptual window within which sensory information can be integrated (Bruno, 2011). It was further suggested that this arrangement makes cortical neurons sensitive to synchronized thalamic input (Bruno, 2011; Bruno and Sakmann, 2006; Wang et al., 2010). In the motor pathways, the same FFI architecture can thus have similar effects on single cell activity but very different behavioral consequences. We previously posited that the excitation/inhibition interplay triggered by the CTC input plays a role in generating the firing transient at the onset of movement (Nashef et al., 2018a). Consistent with this hypothesis, blocking the cerebellar outflow by dentate cooling (Hore and Flament, 1988) or high frequency stimulation in the SCP (Nashef et al., 2019) abolishes this transient, particularly in cortical cells that integrate CTC input. The loss of this transient was accompanied by ataxic movements. These results suggest that thalamo-cortical FFI may play essentially the same role in the temporal organization of activity in both the motor and sensory areas.

The direct and potent contact of inhibitory cells by the CTC system may have additional consequences beyond the temporal organization of cortical activity. Noninvasive cerebellar stimulation in humans (using TMS or tDCS) produces a general inhibition of M1 termed CBI (Ugawa et al., 1991; Ugawa et al., 1995), which is correlated with motor learning (Schlerf et al., 2012). Since the CTC is an excitatory pathway, CBI has been attributed to activation of inhibitory Purkinje cells (Celnik, 2015). Our findings may revise and extend this hypothesis by indicating that $\mathrm{CBI}$ could be related, at least in part, to the CTC activation of motor cortical inhibitory interneurons.

Calcium-permeable AMPA receptors makes TC synapses not only particularly strong, but also prone to plastic changes (Isaac et al., 2007; Plant et al., 2006). This could explain the fact that during motor learning TC input is often modified (Biane et al., 2016), and that the $\mathrm{CBI}$ is correlated with motor learning. Interestingly, the concept of learning via changes in inhibitory connections has been supported by recent theoretical models (Haga and Fukai, 2019; Luz and Shamir, 2012; Mongillo et al., 2018; Vogels et al., 2011).

PV interneuron steer cortical activity and motor output. Cortical PV interneurons are commonly assigned different functional roles. In sensory areas, PV cells are implicated in shaping the tuning of nearby pyramidal cell responses (Atallah et al., 
2012; Hofer et al., 2011; Kerlin et al., 2010; Merchant et al., 2008; Sohya et al., 2007). These cells have also been implicated in maintaining the excitation-inhibition balance needed to regulate cortical activity (Isomura et al., 2009; Xue et al., 2014). Our findings suggest that the PV cells of the motor cortex play a specific role in the temporal organization of neural activity and hence motor behavior. We found that around movement onset, PV cells are recruited before pyramidal cells, probably due to the stronger input PV cells receive from the CTC system. Similar findings were reported for PV neurons in sensory areas (Yu et al., 2019) and in the motor cortex of rodents making spontaneous reaches (Estebanez et al., 2017). However, the time lag between response onset for the two cells types reported here ( $\sim 0 \mathrm{~ms})$ was much larger than in the somatosensory cortex (a few ms). This discrepancy could reflect different properties of both the thalamic volley (e.g., more slowly ramping thalamic activity could produce a larger PV-to-pyramidal time lag) and the cell properties of the PV neurons in different cortical areas (Tasic et al., 2018), such as TC synaptic efficacy, threshold for activation, etc. From a functional perspective, the short PV-to-Pyr time lag found in sensory areas might be optimal for imposing a fast perceptual time window (Bruno, 2011), whereas the longer time-lag found in the motor cortex is more appropriate for timing movements that involve longer time scales.

The functional implications of this early onset of inhibitory signals remain unclear. It is possible that the temporal order of events, together with the enhanced PV-to-Pyr synchrony of SCP-responsive cells, are important for the appropriate onset of movement. At any given point in time, cortical cells integrate a multitude of converging inputs, not all of which are related to the ensuing movement. Thus, early inhibition could suppress these inputs and allow for the subsequent, movementrelated TC excitation to more effectively recruit downstream elements. In this manner, the gain of the thalamic input is enhanced, despite its modest synaptic weight. If true, this arrangement predicts that in the absence of CTC drive, taskrelated activity of $\mathrm{PV}$ and pyramidal cells will overlap in time, reaction time will increase (since it will take more time and effort for activity to produce movement) and the neural and behavioral variability at movement onset will increase (since the initial suppressive effect of PV neurons will not be available). 
422 The early onset of PV cells puts them in an excellent position to steer movements,

423 but the finding that these cells are more strongly correlated with kinematic

424 parameters (i.e., velocity) seems counterintuitive, since pyramidal cells are the

425 output stage of the motor cortex that transmit signals to downstream elements. The

426 stronger synaptic input to PV neurons may make them more easily driven by

427 thalamic input; hence, task-related activity may be more homogeneous across the

428 population of PV cells. In contrast, pyramidal cells are more diverse and their activity

429 is composed of a mixture of multiple (high- and low-level) parameters. As such,

430 although the population activity of pyramidal cells may be strongly correlated with

431 kinematic parameters and drive movements, this relationship is weaker for the

432 activity of single Pyr cells than for single PV cells.

\section{Summary}

434 We report a novel dissection of motor cortical circuitry that integrates cerebellar input 435 during motor behavior. By combining electrophysiological and pharmacological tools, 436 we identified PV and pyramidal neurons in the motor cortex of behaving monkeys.

437 We showed that the two groups of cells have different firing properties (consistent 438 with data from previous studies) but also different patterns of task-related activity.

439 The data indicate that thalamic input in the motor cortex of behaving primates

440 triggers feedforward inhibition, similar to observations in sensory systems. In parallel, 441 we found that PV cells fire earlier than pyramidal cells at movement onset, consistent 442 with the dominant role of cerebellar signals (mediated through the thalamus) at this 443 time. In addition, we found that PV cells are related to task parameters in a similar or 444 stronger manner than pyramidal cells. We therefore suggest that PV neurons are important for prioritizing cerebellar signals at movement onset through the early suppression of competing uncorrelated firing. In this way, these cells act to improve the signal-to-noise ratio for thalamic input, which otherwise might be too weak to 448 properly activate the relevant effectors. The strong thalamic drive to PV cells makes 449 them a more coherent group of neurons (compared to pyramidal cells) and enhances 450 their correlation with kinematic parameters as compared to pyramidal cells. Finally, we suggest that although the operational principle of FFI in general and PV neurons in particular is similar across cortical regions and species, the temporal properties of

453 the motor cortical circuity are tuned to controlling movement timing, rather than integrating percepts as in the sensory cortices. 
Nashef et al. 2020

455

456 This work was funded by the Israel Science Foundation (ISF-1801/18) the Jerusalem

457 Brain Center (to AN) and the generous support of the Baruch Foundation (to YP).

458

459

460 YP and SIP conceived the study. AN performed the experiments and analyzed the

461 data, YP, AN and SIP wrote the manuscript.

462

463

464 The authors declare no competing interest. 
Nashef et al. 2020

A

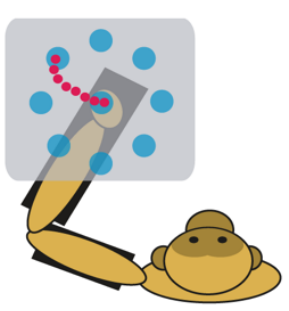

B

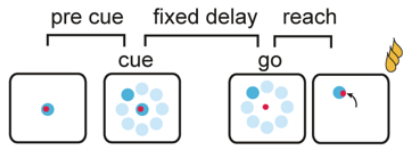

C

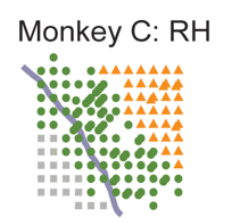

Monkey M: RH

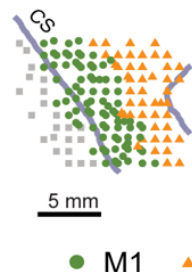

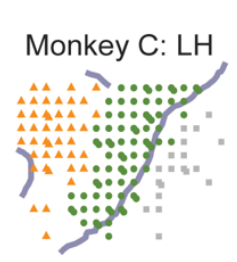

Monkey P: RH

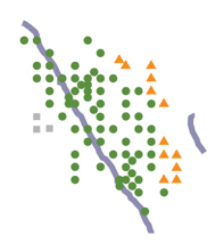

467

468

469

470

471

472

473

474

475

476

477

478

479

480

481

482

483

484

485

\section{Figure 1: Experimental Design.}

(A) Schematic illustration of the experimental setup. Monkeys were trained to wear an exoskeleton and control a cursor that appeared on a horizontally positioned screen. The movement of the monkeys was constrained to planar, shoulder-elbow reaching movements.

(B) Behavioral paradigm. The sequence of events composing a single trial included a pre-cue period, target onset (where 1 of 8 equally distributed targets appeared), a delay period, and a go signal, after which the monkey had to acquire the target within a predefined movement time. Correct performance resulted in a reward (a drop of applesauce).

(C) Cortical maps of recorded sites obtained from monkey $\mathrm{C}$ (right hemisphere, $\mathrm{RH}$; left hemisphere, $\mathrm{LH}$ ) and monkeys $\mathrm{M}$ and $\mathrm{P}$ (right hemisphere only). CS, central sulcus. Scale bar: $5 \mathrm{~mm}$. Green circles: sites in the primary motor cortex (M1). Orange triangles: sites in premotor cortex (PM).

(D) Stimulating electrode was chronically implanted in the cerebellar-thalamo-cortical (CTC). Three-barrel pipette with a tungsten core was used to record and apply drugs iontophoretically in the motor cortex while monkeys performed the task. 
Nashef et al. 2020

A

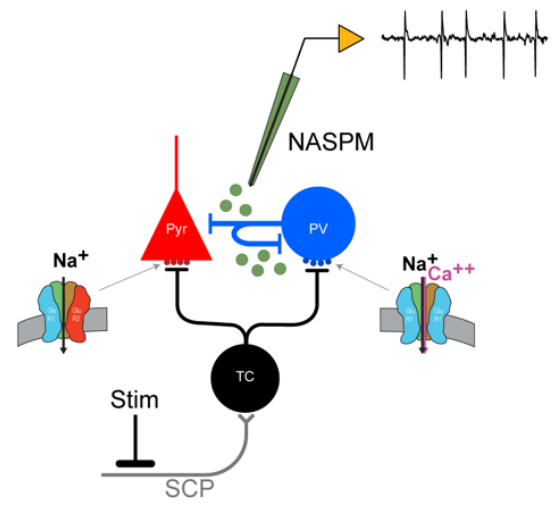

C

Pre-cue firing

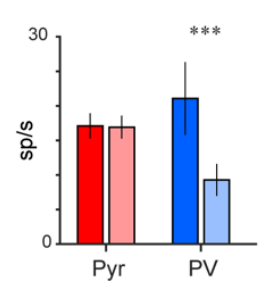

$\mathrm{D}$

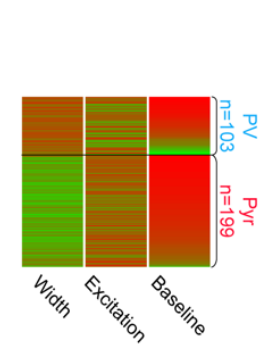

Amplitude; SCP

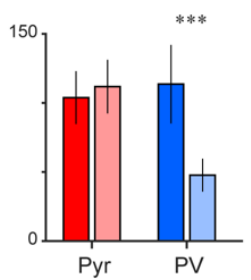

$E$

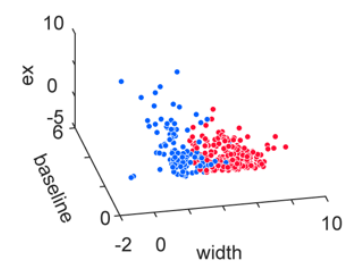

B

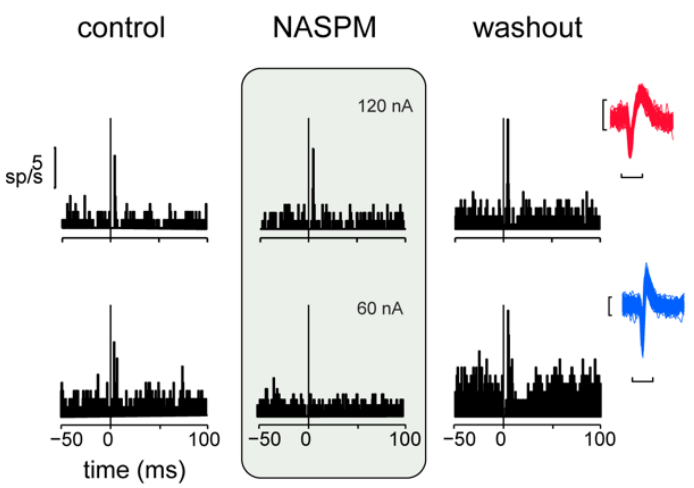

Inhibition; SCP

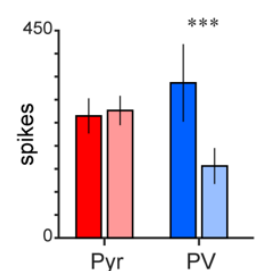

Figure 2: Pyramidal and Parvalbumin neurons identification.

(A) Experimental design for iontophoresis testing. Ascending axons of thalamocortical (TC) neurons make contact with parvalbumin (PV) and pyramidal (Pyr) neurons, the synapse between the TC fibers and PV neurons is mediated by GluR2-lacking AMPA receptors which are $\mathrm{Ca}^{+2}$ permeable. A triple-barrel pipette was filled with 1-Naphthyl acetyl spermine trihydrochloride (NASPM) which is a specific blocker of GluR2-lacking AMPA receptors. During recordings we stimulated the CTC via a stimulation electrode that was chronically implanted in the superior cerebellar peduncle (SCP). We tested the synaptic contact by studying the modulation of response to stimulation during iontophoresis of NASPM. (B) Example of two cells that responded to SCP stimulation and were tested using NASPM. The response is measured using the peri-stimulus time histogram aligned on stimulation onset time $(\mathrm{t}=0)$. Response to SCP stimulation was measured, from left to right, during control trials (left column), during iontophoresis of NASPM (shaded column) and during washout trials (right column). In the presence of NASPM one of the cells (upper panel) maintained responding to SCP stimulation but the second cell (lower panel) stopped responding. Based on these properties we defined the cell on the upper row as a Pyramidal cell and the cell on the lower row as a PV cell. The action potential waveforms are shown as well (right) for the two cells by super-positioning 50 randomly selected waveforms emitted by the Pyramidal (red) 
and PV (blue) cells. (Response: scale bar- $5 \mathrm{sp} / \mathrm{s}$; Waveform: vertical scale bar- 50 $\mu \mathrm{V}$, horizontal scale bar- $1 \mathrm{~ms}$ ).

(C) Differences in firing properties between Pyramidal (red, $n=42$ ) and PV (blue, $\mathrm{n}=16$ ) cells in control trials (dark hues) and in the presence of NASPM (light hues). From left to right: Baseline of activity when no stimulation was applied (Pyr: 17.1 \pm 1.8 to $16.9 \pm 1.7$; PV: $21.1 \pm 3.6$ to $9.3 \pm 2.4 \mathrm{sp} / \mathrm{s}$ ); Amplitude of response to SCP stimulation (mean \pm s.e.m.; control to NASPM; Pyr: $103.8 \pm 19.1$ to $111.8 \pm 19.4$; PV: $113.6 \pm 23.1$ to $47.7 \pm 16.1 \mathrm{sp} / \mathrm{s}$ ); Area under the curve of the excitation period of the SCP response (Pyr: $264.8 \pm 38.1$ to $276.6 \pm 31.8$; PV: $336.5 \pm 57.7$ to $156.1 \pm 42.4$ spikes); Area under the curve of the inhibition period of the SCP response (Pyr: $-487 \pm 53.9$ to 466.2 \pm 49.4 ; PV: $-518 \pm 58.3$ to $-256.7 \pm 44.3$ spikes). ${ }^{* * *} p<0.001$; Wilcoxon's sign-rank. (D) Classification of entire population of SCP-responsive cells $(n=302)$. Three features that we considered for cell classification (Width: width of waveform computed from trough to peak; Excitation: area under the early positive part of the SCP-evoked response; Baseline- background activity when no stimulation was applied). The color scale runs from red (low values) to green (high values) and reflects the differences between the two cell classes. Black horizontal line distinguishes between the Pyramidal (bottom; $n=199$ cells) and PV (top; $n=103$ cells) populations.

(E) A 3-dimensional scatter plot of the three features used for classification. Pyramidal cells are shown as red circles, and PV cells as blue circles. An RCA algorithm (Bar-Hillel et al., 2003; Shental et al., 2002) was used here to optimize the differences between the cell classes.

(F) Comparison between mean clustering score obtained for the data (arrow; score $=1.68$ ) and the distribution of values obtained for shuffled data (bars; $1.01 \pm 0.0003 ; p<<0.001)$. The score was calculated as the distance of each point to the center of the other cluster over its distance to the center of its corresponding cluster.

(E) Comparison between the mean clustering score calculated for the data (arrow; score $=1.68$ ) and the values obtained when using a Gaussian clustering method on $80 \%$ of the neurons ( $\mathrm{n}=242$ neurons) without using the pre-training population of neurons (identified using iontophoresis) as was done here (bars; $1.4 \pm 0.001$; $\mathrm{p}=0.012$ ).

See also Figure S1. 
A
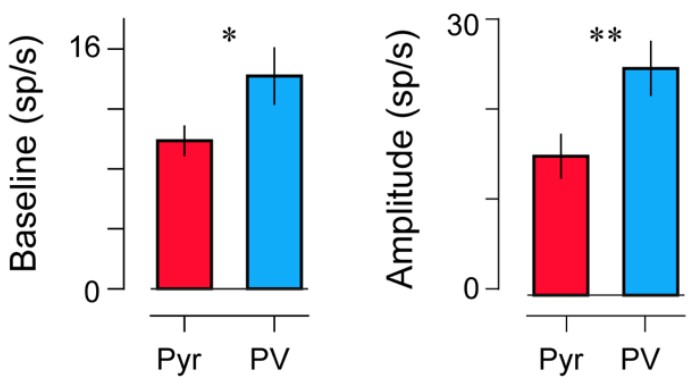

D

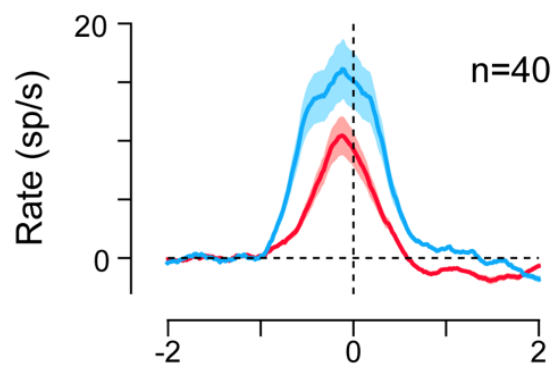

Time (s)

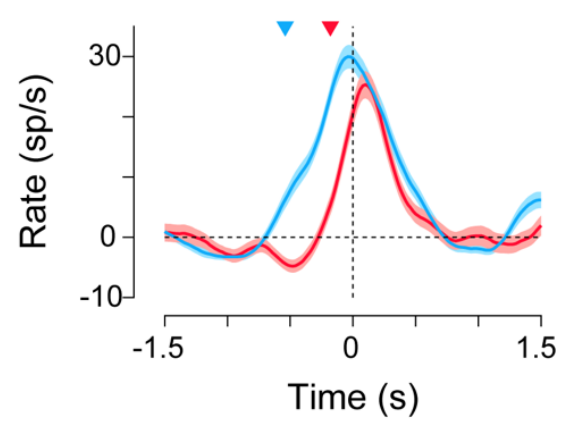

E

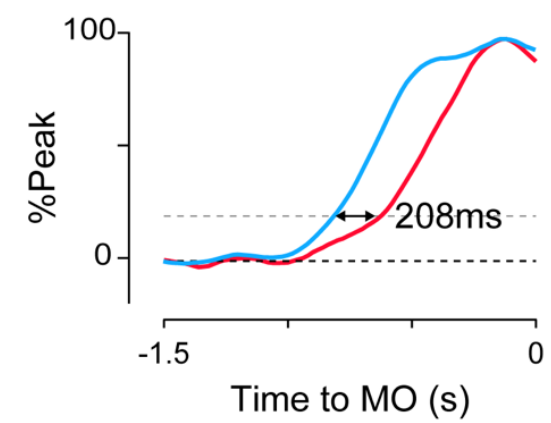

542

543

544

545

546

547

548

549

550

551

552

553

554

555

556

557

558

559

560

561

Figure 3: Task-related activity of simultaneously recorded PV and pyramidal cells during reaching.

(A) Baseline activity computed for simultaneously recorded Pyramidal (red) and PV (blue) cells during a 1 second time window spanning from -2 to $-1 \mathrm{~s}$ relative to movement onset, when no movement took place ( $\mathrm{n}=40$ pairs; Pyr: $9.9 \pm 1.02$; PV: $14.2 \pm 1.9 \mathrm{sp} / \mathrm{s}$; Wilcoxon's sign rank: $p<0.05$ ).

(B) Response amplitude measured as the maximal firing rate around movement onset for the same group of cells as in A. (Pyr: 25.3 \pm 2.8 ; PV: $39.4 \pm 3.8 \mathrm{sp} / \mathrm{s}$; $\mathrm{p}<0.003)$.

(C) Example of task-related activity around movement onset for Pyr (red) and PV (blue) cells that were recorded simultaneously. Shaded area depicts the standard error of the mean and arrowheads highlight the response onset time calculated for each neuron (Pyr onset=-180 ms; PV onset=-540 ms).

(D) Average ( \pm s.e.m) task-related activity of simultaneously recorded pyramidal and PV cells. Activity is aligned on movement onset and baseline activity was subtracted. (E) Normalized neural activity obtained by dividing by peak firing rate around movement onset and expressed in percentages. Difference between average profiles was measured to be 208 ms which was statistically significant (Wilcoxon's sign-rank; $p<0.05 n=40$ cell pairs). 
Nashef et al. 2020

A

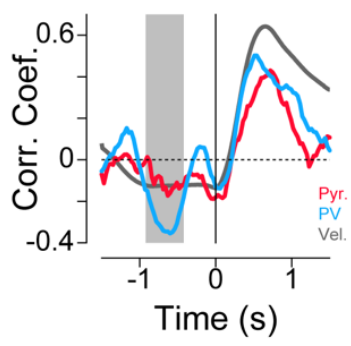

D

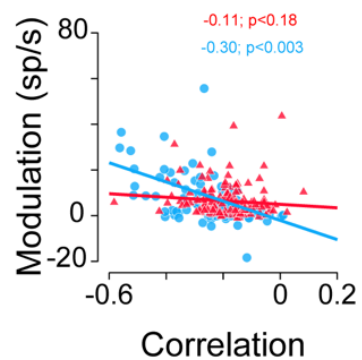

B

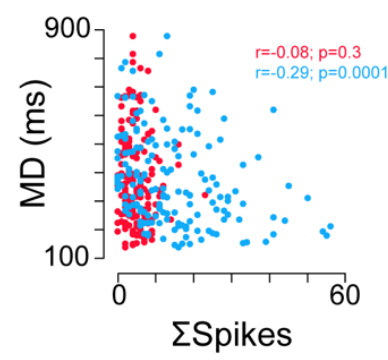

$E$

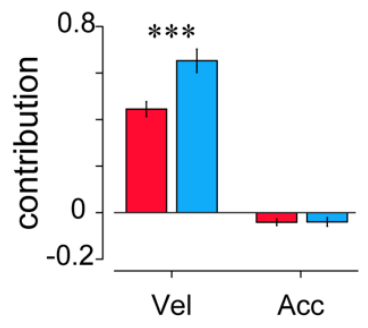

C

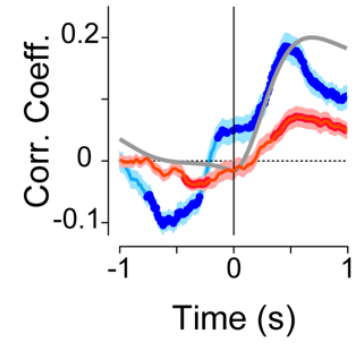

$\mathrm{F}$

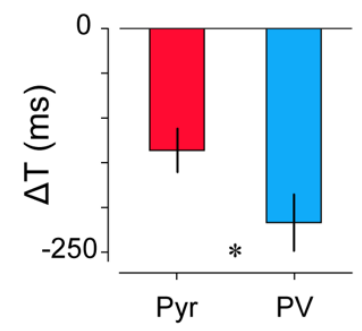

563

564

565

566

567

568

569

570

571

572

573

574

575

576

577

578

579

580

581

582

583

584

585

586

587

588

589

590

591

592

593

Figure 4: Cell-specific neural-behavioral correlation during reaching.

(A) Example of a time-resolved correlation computed for two cells (red: pyramidal; blue: PV) between trial-to-trial firing rates and corresponding movement times. Correlation values were obtained by measuring single trial firing rate in a $500 \mathrm{~ms}$ sliding windows and correlating it with movement time. Correlation functions were aligned on movement onset. Gray box shows the time window from which the firing values were taken in panel (B).

(B) Scatter plot of the movement times (y-axis) against single trial spike counts ( $x$ axis) of each cell (red-Pyr firing and blue-PV firing) as measured for the highlighted gray box shown in A. (Pyr-MT correlation: $\rho=-0.08, p=0.3$; PV-MT correlation: $\rho=-$ $0.29, p=0.0001$; Pearson's correlation).

(C) Average time-resolved correlation function between firing rate and movement time computed for pyramidal (red) and PV (blue) cells. Shading area corresponds to the standard error of the mean. Filled circles highlight the time points when the correlation value was significantly different from zero (Wilcoxon's sign-rank; $\mathrm{p}<0.0006$ - Boneferroni's correction for multiple comparisons- $0.01 /(500 / 30) ; 500 \mathrm{~ms}$ is the time window and $30 \mathrm{~ms}$ is the time steps). Gray trace shows the average hand position (distance from the center target) recorded in parallel to the neural data. Time zero corresponds to movement onset.

(D) The effect of firing rate level on firing-to-behavior correlation was estimated by plotting the relations between single cell rate modulation (relative to baseline level) measured at time point of minimal correlation and the correlation coefficient value for Pyr (red triangles) and PV (blue circles) cells. The correlation coefficient between the two parameters were computed and their significance level was estimated (Pyr: $\rho=-$ $0.11, p=0.18$; PV: $\rho=-0.30, p=0.003$; Pearson's correlation). The lines show the linear regression computed for each set of values $\left(R^{2}\right.$ : $\left.P V=0.093 ; P y r=0.012\right)$.

(E) The contribution of velocity (Vel) and acceleration (Acc) to the reconstruction of firing rate (see Methods section for details. Velocity contribution: $P y r=0.45 \pm 0.03$, $P V=0.65 \pm 0.05, p<0.001$; Acceleration contribution: $P V=-0.04 \pm 0.02, P y r=-0.04 \pm 0.01$; Wilcoxon's sign-rank). 
bioRxiv preprint doi: https://doi.org/10.1101/2020.10.20.346775; this version posted October 21, 2020. The copyright holder for this preprint (which was not certified by peer review) is the author/funder, who has granted bioRxiv a license to display the preprint in perpetuity. It is made available under aCC-BY-NC-ND 4.0 International license.

Nashef et al. 2020

594 (I) Mean time shift $(\Delta \mathrm{T})$ required to achieve maximal similarity between actual neural 595 firing and reconstructed firing (PV=-217 $\pm 31.9 \mathrm{~ms}$, Pyr= -134.7 $\pm 24.9 \mathrm{~ms}$; Wilcoxon's 596 sign-rank, $p<0.05)$.

597 See also Figure S2.

598 
Nashef et al. 2020
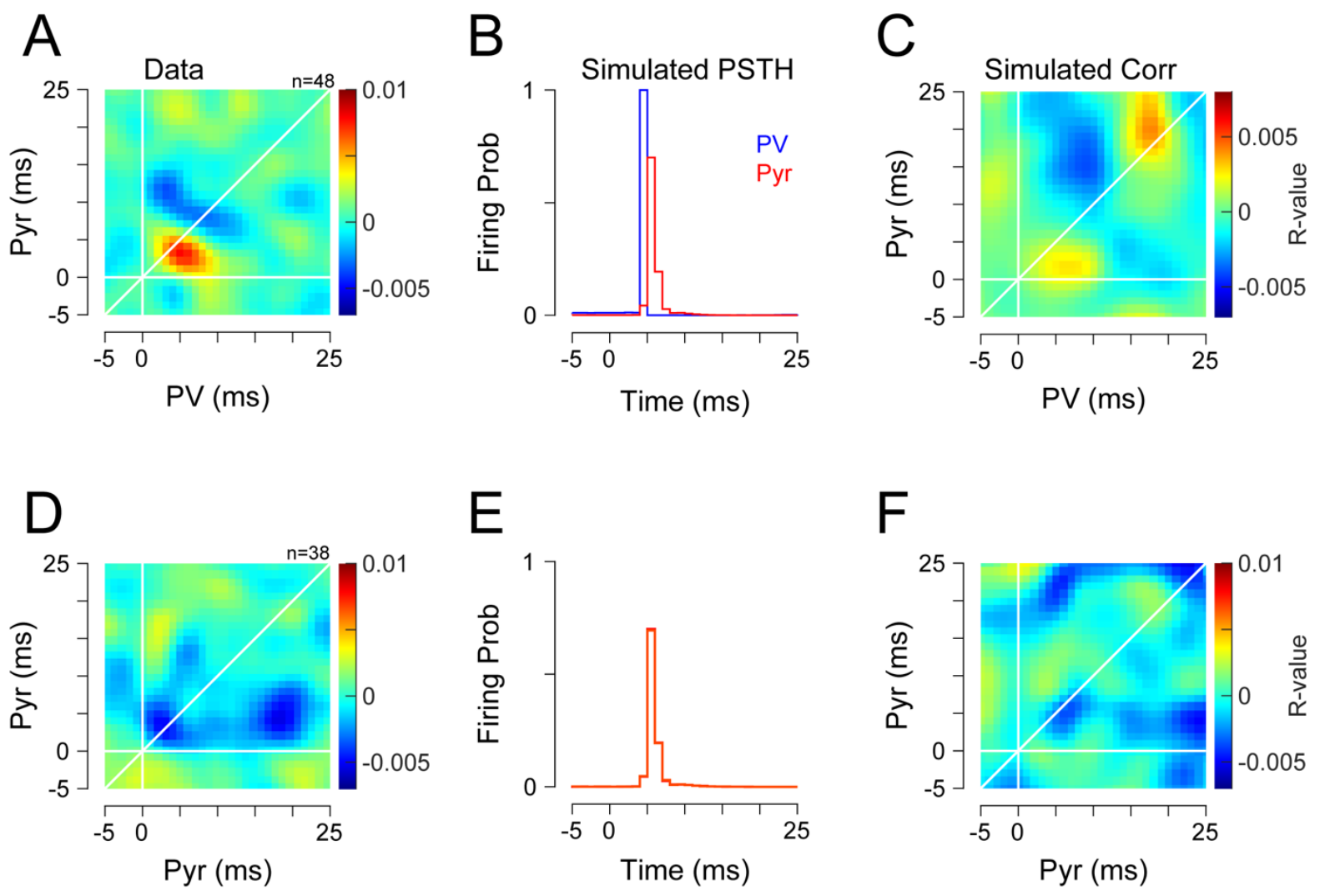

600 Figure 5: Joint Peri-stimulus time histogram of PV and pyramidal pairs

601 (A) Average Joint peri-stimulus time histogram (jPSTH) of simultaneously recorded 602 pairs of PV and pyramidal cells $(n=48)$. Time zero is stimulation time. Color scale 603 corresponds to the normalized correlation values (actual counts - expected counts 604 over the standard deviations). Vertical and horizontal white lines indicate the time of 605 the stimulation. Diagonal line corresponds to the zero-lag time between the two cells. 606 (B) "Stimulus-triggered response" of simulated cell pairs ( $n=100$ pairs; 400 trials) as 607 obtained from a model of a feed-forward inhibition circuit. Responses were aligned 608 on thalamic spike times $(\mathrm{t}=0)$.

609 (C) Average jPSTH computed between simulated pairs of PV and Pyramidal cells 610 that resulted from the simulated circuitry as shown in $B$.

611 (D-F) same as A-C but for pairs of pyramidal cells. The number of recorded pairs used to compute (D) was $n=38$ and for $F$ number of simulated pairs was the same as 613 in $C(n=100$ pairs; 400 trials).

614 See also Figure S3. 
Nashef et al. 2020

A
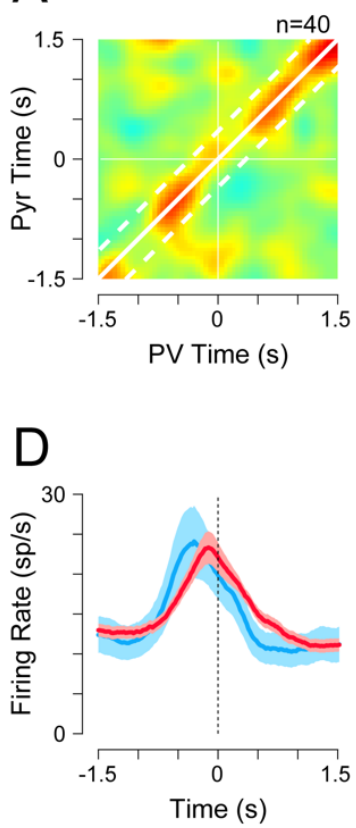

B

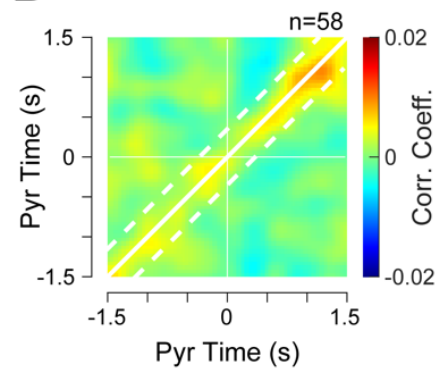

$\mathrm{E}$

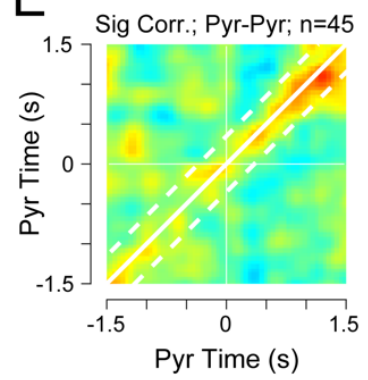

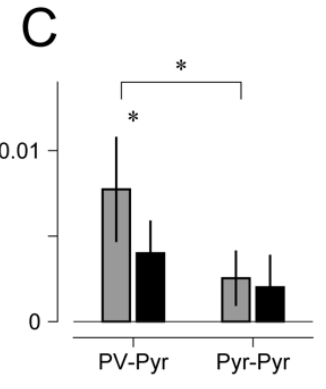

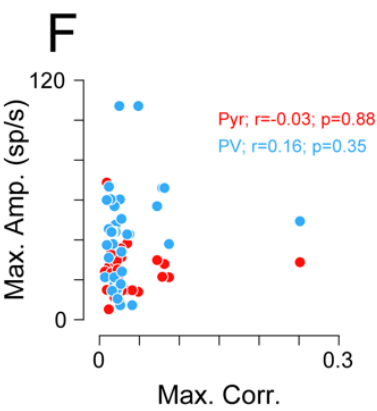

Figure 6: Joint Peri-event time histogram of PV and Pyramidal pairs around movement onset

(A) Average Joint Peri-event time histogram (jPETH) for pairs of PV and pyramidal neurons around movement onset. White horizontal and vertical lines denotes movement onset time $(\mathrm{t}=0)$. Solid diagonal line corresponds to zero inter-unit lag time while dashed lines corresponds to the time around the diagonal within which the average was calculated for panel $\mathrm{C}$. Color scale corresponds to the R-value of the correlation martix ((counts-predicted)/std; $n=40$ pairs). movement (black bars), was calculated during a time window spanning -600 to -100 ms relative to movement onset and -100 to $+400 \mathrm{~ms}$ around the same event. The inter-unit time lags that were considered for computing the averaged correlation were $\pm 350 \mathrm{~ms}$ (shown as diagonal lines in panels $A$ and $B$ ). Each bar represented the mean correlation value computed across pairs and the corresponding s.e.m. (PV-Pyr pairs; $n=40$ pairs; Base: $0.008 \pm 0.003$; Move: $0.004 \pm 0.002$; Wilcoxon's sign-rank test; $p<0.02$; Pyr-Pyr pairs; $n=58$ pairs; Base: $0.0025 \pm 0.001$; Move: 0.002 \pm 0.0019 ; $p=0.72$ ). During pre-movement period (baseline) the baseline correlation between PV-Pyr and Pyr-Pyr pairs was significantly different $(p<0.02)$.

(D) The average movement related activity of PV and a subset of Pyramidal neurons that had a specifically high response amplitude around movement onset $(n=45$ neurons). Responses were aligned and movement onset time and shaded areas show the s.e.m.

(E) Same as B, but for computed for the subset of Pyramidal cells shown in D. . circles) or Pyr (red) neurons (Pyr: $\rho=-0.03, p=0.88$; PV: $\rho=0.16, p=0.35$; Pearson's correlation). 
Nashef et al. 2020

A

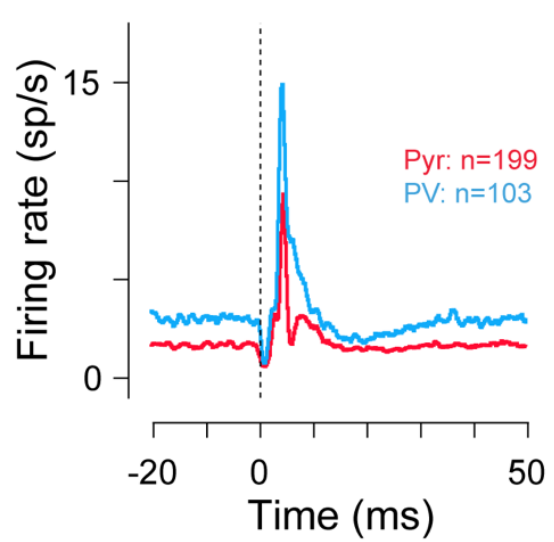

\section{B}

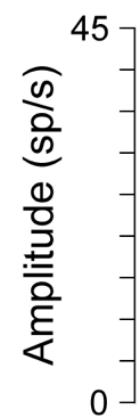

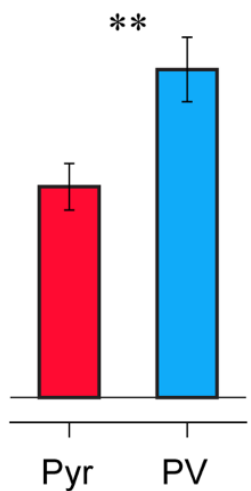

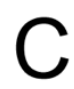

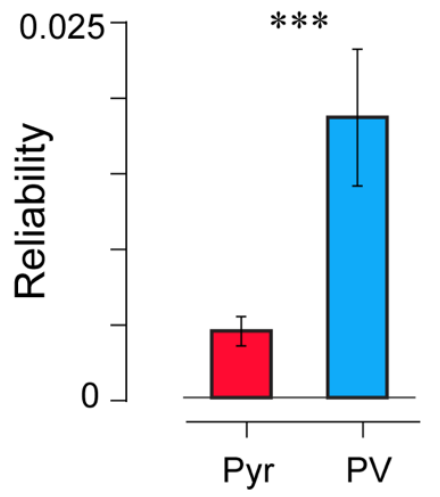

Figure S1: SCP response properties of classified cells, related to Figure 2.

(A) The average response to SCP stimulation of classified Pyr and PV cells. Vertical line corresponds to the stimulation time (Pyr- $n=199$ cells and $P V-n=103$ cells). (B) Comparison of the amplitude of the different classes of cells following SCP stimulation, measured as the maximal firing rate following the stimulation (Pyr: 25.3 \pm 2.8 ; PV: $39.4 \pm 3.9$ sp/s; Wilcoxon's sign-rank; $p<0.005$ ).

(C) Comparison of the response reliability (Wang et al., 2010) of the different classes of cells following SCP stimulation (Pyr: 0.004 \pm 0.0004 ; PV: 0.019 \pm 0.003 ; Wilcoxon's sign-rank; $p<0.001)$. 
A
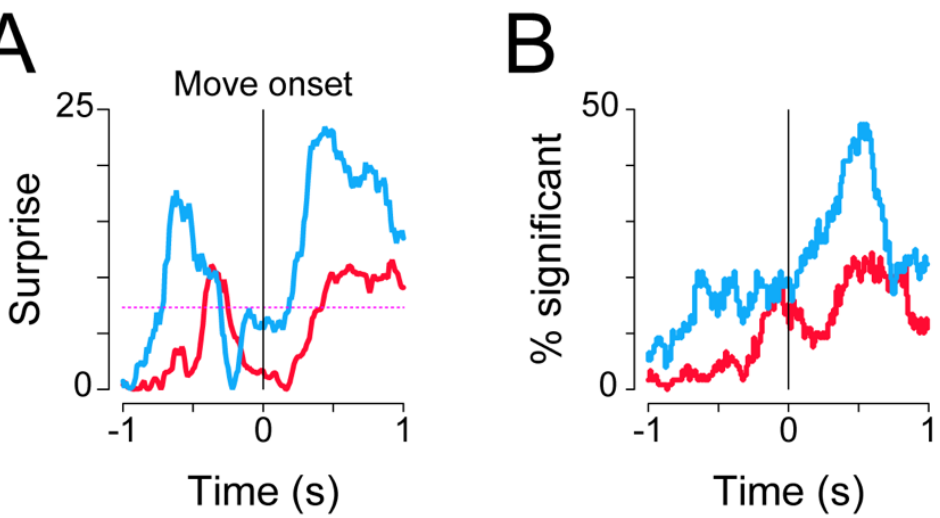

660 Figure S2: Cell-specific neural-behavioral correlation during reaching, related 661 to Figure 4.

662 (A) Mean surprise values $(-\log (p))$ computed for the correlation functions shown in 663 (Figure 4C), dashed line denotes the threshold for determining significance (i.e., 664 surprise value of 7.4).

665 (B) Fraction of cells that exhibited significant correlation between rate and movement 666 time as computed in Figure 4C. 
A

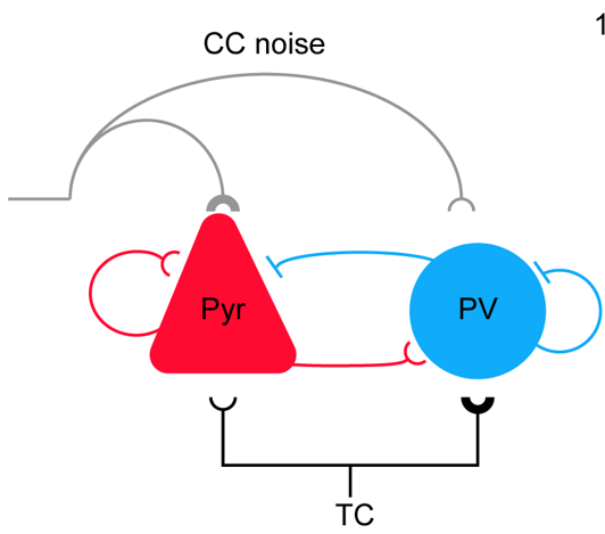

B

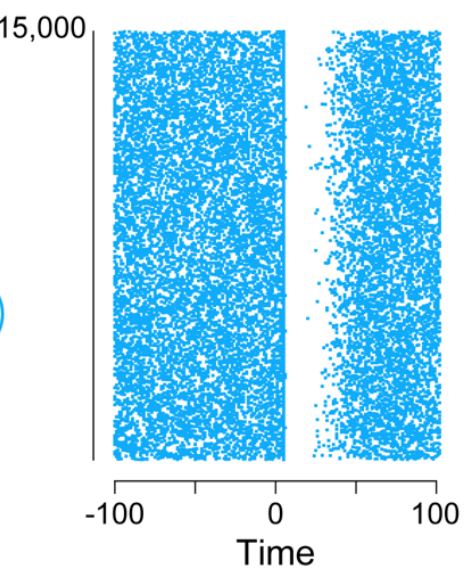

C

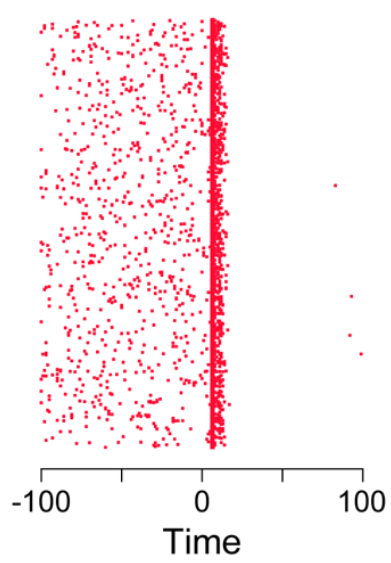

Figure S3: Simulated Feed Forward Inhibition circuitry, related to Figure 5.

670 (A) The synaptic connections that were used to model the feed-forward inhibitory 671 circuity. Both PV and Pyr cells received an excitatory input from thalamocortical 672 neurons (TC)- the input to PV was however 4 times stronger than the input to Pyr 673 neurons. Each cell then contacts the other cell and itself (autoactivation for Pyr and 674 autoinhibition for PV cell). Both cell types also receive a corticocortical noisy input. 675 (B) Raster of PV response to simulated TC input. Each line corresponds to the PV 676 response to one TC spike, and time zero is the TC spike time.

677 (C) Same as B, but for Pyr cell. 
Nashef et al. 2020

A

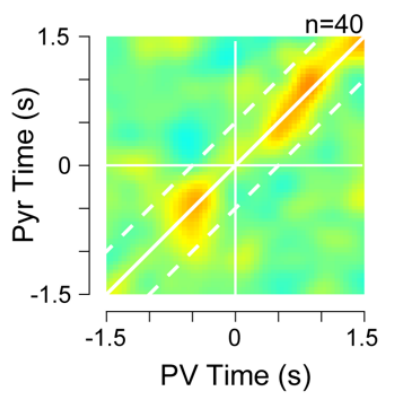

$\mathrm{D}$

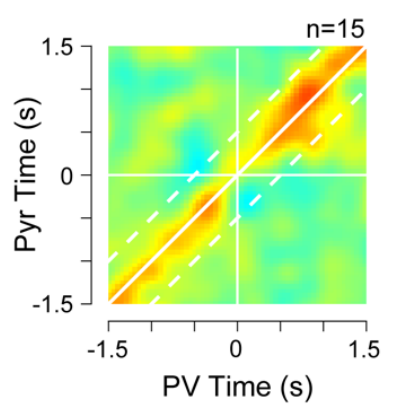

B

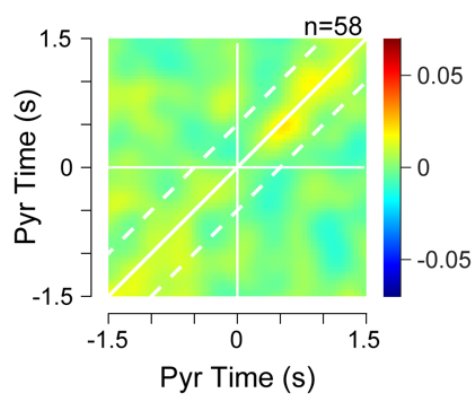

E

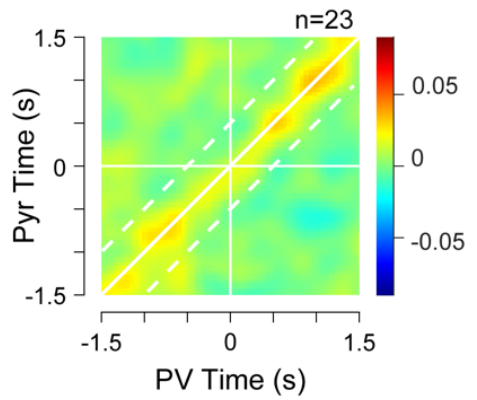

C

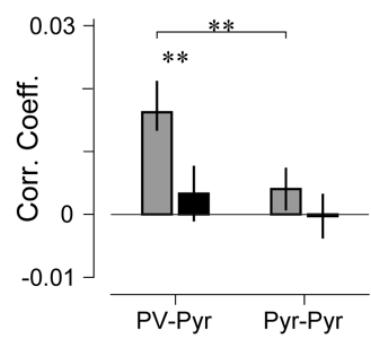

$\mathrm{F}$

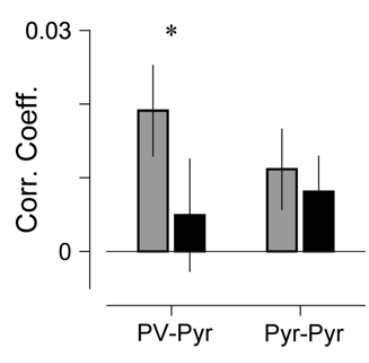

Figure S4: Joint Peri-event time histogram of PV and Pyramidal pairs around movement onset, related to Figure 6

681

(A) Average jPETH for pairs of PV and pyramidal neurons around movement onset.

682 White horizontal and vertical lines denotes movement onset time $(\mathrm{t}=0)$. Solid

683 diagonal line corresponds to zero inter-unit lag time while dashed lines corresponds to the time around the diagonal within which the average was calculated for panel C. Color scale corresponds to the R-value of the correlation martix (countspredicted)/std; $n=40$ pairs). The jPSTH was aligned around the PD of one unit of the pair randomly.

(B) Same as (A) for pyramidal-pyramidal pairs ( $n=58$ pairs).

(C) The average correlation at baseline (pre-movement time, gray bars) and during movement (black bars), was calculated during a time window spanning -600 to -100 ms relative to movement onset and -100 to $+400 \mathrm{~ms}$ around the same event. The inter-unit time lags that were considered for computing the averaged correlation were $\pm 350 \mathrm{~ms}$ (shown as diagonal lines in panels A and B). Each bar represented the mean correlation value computed across pairs and the corresponding s.e.m. (PV-Pyr pairs; $n=40$ pairs; Base: $0.017 \pm 0.004$; Move: $0.003 \pm 0.004$; Wilcoxon's sign-rank test; $p<0.006$; Pyr-Pyr pairs; $n=58$ pairs; Base: $0.004 \pm 0.003$; Move: $-0.0003 \pm 0.004$; $p=0.29$ ). During pre-movement period (baseline) the baseline correlation between PV-Pyr and Pyr-Pyr pairs was significantly different $(p<0.01)$.

(D) Same as (A) but for pairs of units with similar PD- pairs that have similar or neighboring PD $(n=15)$.

(E) Same as (A) for pyramidal-pyramidal pairs, that have similar PD $(n=23)$. $n=23$ pairs; Base: $0.01 \pm 0.006$; Move: $0.008 \pm 0.005 ; p=0$. 95). 


\section{STAR METHODS}

\begin{tabular}{|c|c|c|}
\hline \multicolumn{3}{|c|}{ RESOURCE TABLE } \\
\hline $\begin{array}{l}\text { Reagent or } \\
\text { Resource }\end{array}$ & Source & Identifier \\
\hline \multicolumn{3}{|c|}{ Experimental model: Organisms/strains } \\
\hline Macaca fascicularis & $\begin{array}{l}\text { The Hebrew } \\
\text { University }\end{array}$ & N/A \\
\hline \multicolumn{3}{|c|}{ Software and Algorithms } \\
\hline MATLAB 2016b & Mathworks & https://www.mathworks.com/ \\
\hline \multicolumn{3}{|l|}{ Drugs } \\
\hline $\begin{array}{l}\text { 1-Naphthylacetyl } \\
\text { spermine } \\
\text { trihydrochloride }\end{array}$ & Sigma Aldrich & $\begin{array}{l}\text { hhttps://www.sigmaaldrich.com/united- } \\
\text { states.html }\end{array}$ \\
\hline \multicolumn{3}{|l|}{ Other } \\
\hline NSEX-100 & NSEX-100 & NSEX-100 \\
\hline
\end{tabular}

\section{CONTACT FOR RESOURCE SHARING}

Further information and requests for resources and reagents should be directed to and will be fulfilled by the lead contact, Yifat Prut (yifatpr@ekmd.huji.ac.il).

\section{EXPERIMENTAL MODEL AND SUBJECT DETAILS}

This study was performed on three adult female monkeys (Macaca fascicularis, weight $4.5-8 \mathrm{~kg}$ ). The care and surgical procedures of the subjects were in accordance with the Hebrew University Guidelines for the Use and Care of Laboratory Animals in Research, supervised by the Institutional Committee for Animal Care and Use.

\section{METHOD DETAILS Behavioral task}

Data were obtained from three Macaca fascicularis monkeys (females, $4.5-8 \mathrm{Kg}$ ). Primate care and surgical procedures were in accordance with the Hebrew University Guidelines for the Use and Care of Laboratory Animals in Research, supervised by the Institutional Committee for Animal Care and Use. The behavioral task used here was described in our previous study (Nashef et al., 2019). Briefly, the three monkeys were trained to sit in a primate chair, wear a unilateral exoskeleton (KINARM, BKIN technologies), and perform an arm reaching task. The exoskeleton constrained the arm to move in a horizontal plane at the level of the shoulder, and allowed free movement about the elbow and shoulder joints in that plane. The end 
point coordinates of the exoskeleton (i.e., the monkey's hand position) controlled the location of a cursor on a horizontally positioned screen in front of the animal (Figure $1 \mathrm{~A})$. In this task, the monkeys were instructed to move the arm to locate a cursor within a central target. After $500 \mathrm{~ms}$, a peripheral target (one of 8 evenly distributed targets) appeared. After a fixed delay time of 300-750 ms the central target disappeared ("GO" signal), cuing the monkey to reach to the visible peripheral target. If the monkey moved the cursor to the correct target within the predefined time limits it was rewarded with a drop of applesauce. To encourage the monkey to predict the timing of the "go" signal, we limited the total time it had to reach the peripheral target after the GO signal to $500 \mathrm{~ms}$ and inserted a $200 \mathrm{~ms}$ grace period before the GO signal. Onset of movement within this time frame did not abort the trial.

After training was completed, a recording chamber $(21 \times 21 \mathrm{~mm})$ was attached to the monkeys' skull above the hand-related area of the motor cortex in a surgical procedure under general anesthesia. After a recovery and re-training period, we recorded motor cortical activity extracellularly by advancing electrodes through the chamber in daily recording sessions.

\section{Insertion of stimulating electrode into the superior cerebellar peduncle (SCP).}

To insert a chronic stimulating electrode into the SCP ipsilateral to the trained arm, we implanted a small chamber above the cerebellum (based on a macaque neuroatlas) and used a post-surgery MRI to plan an electrode trajectory to the SCP. A bipolar concentric electrode (NSEX100, David Kopf Instruments, impedance range of $30-60 \mathrm{k} \Omega$ ) was positioned in the peduncle through the chamber. Evoked intracortical responses to stimulation through the electrode were used to verify its location before gluing the electrode to the chamber (Nashef et al., 2019; Nashef et al., 2018a; Nashef et al., 2018b; Ruach et al., 2015).

\section{SCP stimulation protocol.}

Biphasic (200 $\mu$ s each phase) stimulation pulses were applied to the SCP electrode while the monkey performed the task and cortical activity was recorded. A single set of stimuli consisted of about 200 stimuli that were delivered at $3 \mathrm{~Hz}$ at a fixed intensity (ranging from 50 to $300 \mu \mathrm{A}$ ).

\section{Recording}

Recordings were obtained using one of two methods:

1- Iontophoresis sessions: For a full description of the method, see (Thiele et al., 2006). During the iontophoresis sessions, three-barrel pipettes were inserted through the chamber to different cortical sites, mostly in the primary motor cortex (M1) which was defined based on MRI scans, their relation to anatomical landmarks (i.e., the central and arcuate sulci) and threshold for motor response. The central barrel in the pipette contained a tungsten electrode (impedance $300-800 \mathrm{k} \Omega$ at $1,000 \mathrm{~Hz}$ ) for extracellular recording. Before each recording session, the two peripheral barrels were filled with NASPM (or glutamate) using polyamide hypodermal injection tubing (purchased from World Precision Instruments, Ltd.), and a bare tungsten wire was 
bioRxiv preprint doi: https://doi.org/10.1101/2020.10.20.346775; this version posted October $21,2020$. The copyright holder for this preprint (which was not certified by peer review) is the author/funder, who has granted bioRxiv a license to display the preprint in perpetuity. It is made available under aCC-BY-NC-ND 4.0 International license.

Nashef et al. 2020

inserted into each barrel and held in place using a drop of glue. The signal obtained from each electrode was amplified $\left(\times 10^{4}\right)$, bandpass-filtered $(300-6,000 \mathrm{~Hz})$, digitized $(32 \mathrm{kHz})$, and saved to disk. NASPM and glutamate were ejected from the pipette by applying a positive or negative current, respectively, to the tungsten wires (30-200 $n A)$. A - 15 nA retention current of the opposite sign was applied to the wires at other times.

2- Other sessions: Glass coated tungsten electrodes (impedance $300-800 \mathrm{k} \Omega$ at $1,000 \mathrm{~Hz}$ ) were inserted through the chamber to different cortical sites, mostly in $\mathrm{M} 1$. The signal obtained from each electrode was amplified $\left(\times 10^{4}\right)$, bandpass-filtered $(300-6,000 \mathrm{~Hz})$, digitized $(32 \mathrm{kHz})$, and saved to disk. Recordings were made with up to 4 individually moveable electrodes (Flex-MT by Alpha Omega, Nazareth, Israel).

\section{NASPM preparation}

1-Naphthylacetyl spermine trihydrochloride (NASPM, Sigma-Aldrich; molecular weight $=479.91 \mathrm{~g} / \mathrm{mol}$ ) was used for blocking GluR2-lacking AMPA mediated receptors on parvalbumin (PV) cells. Preparation of NASPM started with dissolving the powder $(5 \mathrm{mg})$ into $2 \mathrm{cc}$ of saline (concentration $5 \mathrm{mM}$ ). The solution was kept at $80 \mathrm{C}^{\circ}$ and warmed before use. For injection, we diluted the solution further with saline to obtain a final concentration of $1 \mathrm{mM}$ and $\mathrm{pH}$ of 5-6; the final solution was not kept for more than 7 days after preparation. We recorded responses of cortical neurons to glutamate iontophoresis in one session per monkey for control. Here, 100 $\mathrm{mg}$ of L-glutamate was dissolved in $6.796 \mathrm{ml}$ of artificial CSF and titrated with $\mathrm{HCO}_{3}{ }^{-}$ to increase the $\mathrm{pH}$ to between 7.4-7.8.

\section{QUANTIFICATION AND STATISTICAL ANALYSIS}

\section{Cell classification}

We combined naïve Bayesian and support-vector machine (SVM)(Cortes and Vapnik, 1995) classifiers to identify PV and pyramidal cells. First, we trained the classifier with data from cells that were tested using NASPM $(n=58)$. For each cell we calculated several features to guide the classification: width of action potential, amplitude of waveform (peak-trough), slope of waveform (amplitude/width), decay of waveform after peak, peak-to-trough (peak/trough), amplitude to SCP stimulation, gain after SCP stimulation, area of excitatory response following SCP stimulation, area of inhibitory response following SCP stimulation and pre-stimulus firing. This step was carried out to find the parameters that would yield maximal performance. We assessed the performance of the classifier for all the combinations of the 9 features mentioned (overall, 511 different combinations). For each combination a relevant-component analysis (RCA) was performed over the data, to optimize the distance between the two cell classes(Bar-Hillel et al., 2003; Shental et al., 2002). Next, we ran the classifier 100 times for each of the cells in the training set $(n=58)$, where at each iteration a random $60 \%$ selection of the remaining cells $(n=34)$ were considered. In practice this means that in each iteration the classifier was retrained with the randomly selected subset of 34 cells in order to minimize the effect of noisy labels. After the 100 iterations, the most common label in both classifiers was considered to be true. For instance, if the naïve Bayesian labeled 70 cases (out of 100 iterations) as PV and SVM labeled 55 cases as Pyr, the naïve Bayesian result, 
bioRxiv preprint doi: https://doi.org/10.1101/2020.10.20.346775; this version posted October $21,2020$. The copyright holder for this preprint (which was not certified by peer review) is the author/funder, who has granted bioRxiv a license to display the preprint in perpetuity. It is made available under aCC-BY-NC-ND 4.0 International license.

Nashef et al. 2020

826

827

828

829

830

831

832

833

834

835

836

837

838

839

840

841

842

843

844

845

846

847

848

849

850

851

852

853

854

855

856

857

858

859

860

861

862

863

864

865

866

867

868

869

870

871

872

873

874

875

$\mathrm{PV}$, was taken as the final classification. We found that width, pre-stimulus firing activity and excitation following SCP stimulation were the most important features in distinguishing between the two subpopulations providing an accuracy of $75 \%$ in classification ( $76 \%$ of the PV and $75 \%$ of Pyr), based on the cells tested with NASPM. The classifier was then applied to the cells that were not recorded during NASPM application (the unknown labels), in the same way.

\section{Data analysis}

Stimulus-evoked responses of single neurons. We identified SCP-responsive cortical neurons according to a previously published method (Nashef et al., 2019; Nashef et al., 2018a).

Simulation of a feed-forward inhibition (FFI) circuit. We simulated an FFI circuit using a previously published (Izhikevich, 2003) excitatorylinhibitory (EVI) cortical network (http://izhikevich.org/publications/spikes.htm) with randomized incoming thalamocortical input. To mimic the SCP stimulation, we enhanced the randomized input at time zero. Furthermore, we used parameters that were found in the past for the strength of thalamocortical input on PV and pyramidal neurons (Cruikshank et al., 2007), specifically, the conductance of the thalamic-PV synapse was set to $3 \mathrm{nS}$ and thalamic-Pyr. to $0.7 \mathrm{nS}$, to ensure reliable inhibition in our circuitry. Other well defined parameters were: spiking threshold $=-57 \mathrm{mV}$; resting potential $=-70 \mathrm{mV}$; $\mathrm{T}(\mathrm{Pyr})=2 \mathrm{~ms} ; \mathrm{T}(\mathrm{PV})=10 \mathrm{~ms}$. The intracortical excitatory synapse conductance at $1 \mathrm{nS}$ and the inhibitory at $2 \mathrm{nS}$ (Kremkow et al., 2010). To mimic our experiment, we simulated 400 trials of incoming input for each connected pair, averaged the firing probability and found the jPSTH for these "simultaneous" 400 trials. To insert noise in the response, at each trial we randomized the connectivity strength between the PV cell and the pyramidal cell, and also the auto-connectivity of the individual cell. At the end, for each pair we had two (one PV and one Pyr) matrices of $400 \mathrm{x}$ time, where each row is the firing of the cells around one CTC input. This whole process was performed 50 times, to resemble different pairs and then the average response and average jPSTH were found for these 50 pairs, using bin-by-bin averaging. Task-related response properties of recorded neurons. 1-Background firing- The background firing of each neuron was calculated as the average firing rate when no task-related modulation occurred, in the time window between 2 and 1 seconds before movement onset $(-2$ to $-1 \mathrm{sec})$.

2- Preferred Direction-For each single unit, we computed the tuning function and its preferred direction (PD). The preferred direction was calculated individually for each isolated unit using a resampling method (Shalit et al., 2012) (4,000 repetitions) during the -500 to 500 ms around movement onset.

3- Orientation selectivity index (OSI)-First we calculated the tuning curve of each cell, and then the OSI was defined as $1-r$, where $r$ is the circular variance of the tuning curve (Atallah et al., 2012).

4- Onset time of the task-related activity- To calculate the response onset time of neurons while overcoming their noisy background firing we applied the following steps. First, time of movement onset was defined using the $X Y$-position signals that are continuously recorded in the KINARM setup. A dedicated algorithm identified transitions in the distance of the instantaneous $X Y$ position of the cursor to the center of the central target (Asher et al., 2010). Next, we calculated the average perievent time histogram (PETH, background firing subtracted) for each neuron around movement onset and found the maximal firing rate in the window $\pm 1 \mathrm{sec}$ around movement onset. We then normalized the firing rate by the maximum rate and, 
Nashef et al. 2020

876 starting at the peak time we moved backwards in time until the point when the firing

877 rate dropped below 0.2 (20\% of the peak) for the first time. This was defined as the

878 onset of the movement-related firing.

879

880 Joint Peri-Stimulus Time Histogram. We based our quantification of the joint PSTH

881 on previous work (Aertsen et al., 1989). First, we used the PSTH's of two neurons to

882 calculate the raw JPSTH matrix, in which the $(i, j)$-th bin was the time of coincidence

883 in which one neuron spiked at the $i^{\text {th }}$ bin and the second spiked at the $j^{\text {th }}$ bin. To

884 correct for rate modulation we calculated the PSTH predictor (Aertsen et al., 1989).

885 The predictor matrix is the product of the single neuron PSTHs; i.e., the (i,j)-th bin is

886 equal to PSTH1(i)*PSTH2(j). The final jPSTH was calculated as: jPSTH ${ }^{*}$ redict -

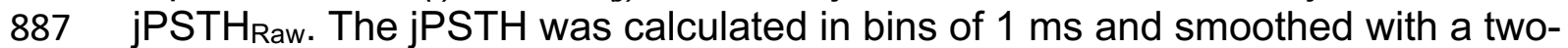

888 dimensional Gaussian window with a SD of $1.5 \mathrm{~ms}$.

889 Neural-Behavioral correlation. We quantified the relationships between neural firing

890 and movement duration, MD by measuring for each cell the time resolved correlation

891 between the vector of spike counts (counted for each trial in a given time window)

892 and the MD found for those trials. For this analysis we only considered cells with at

893 least 20 available trials and a significant tuning. To compute the correlations

894 between spike counts and MD, available trials were first aligned on movement onset

895 (from -1500 to $+1500 \mathrm{~ms}$ around this event) and spike numbers were counted for

896 time windows of $200 \mathrm{~ms}$, advancing in steps of $10 \mathrm{~ms}$. For each window, we

897 computed the correlation coefficient between spike counts and MD. Correlation

898 coefficient were then averaged across all cells to obtain the population-based

899 correlation between rate and MD. The standard error of the mean was then

900 computed per bin for each class of cells. 
Nashef et al. 2020

902

903

904

905

906

907

908

909

910

911

912

913

914

915

916

917

918

919

920

921

922

923

924

925

926

927

928

929

930

931

932

933

934

935

936

937

938

939

940

941

942

943

944

945

946

947

948

\section{REFERENCES}

Aertsen, A.M., Gerstein, G.L., Habib, M.K., and Palm, G. (1989). Dynamics of Neuronal Firing Correlation: Modulation of "Effective Connectivity" Journal of neurophysiology 61.

Asher, I., Zinger, N., Yanai, Y., Israel, Z., and Prut, Y. (2010). Population-based corticospinal interactions in macaques are correlated with visuomotor processing. Cereb Cortex 20, 241252.

Atallah, B.V., Bruns, W., Carandini, M., and Scanziani, M. (2012). Parvalbumin-Expressing Interneurons Linearly Transform Cortical Responses to Visual Stimuli. Neuron 73, 159-170. Babinski, J. (1913). Expose des travaux scientifi que (Paris).

Bar-Gad, I., Ritov, Y.a., Vaadia, E., and Bergman, H. (2001). Failure in identification of overlapping spikes from multiple neuron activity causes artificial correlations. journal of neuroscience methods 107, 1-13.

Bar-Hillel, A., Hertz, T., Shental, N., and Weinshall, D. (2003). Learning distance functions using equivalence relations. International Conference on Machine Learning (ICML), 11-18. Bastian, A., Martin, T., Keating, J., and Thach, W. (1996). Cerebellar ataxia: abnormal control of interaction torques across multiple joints. Journal of neurophysiology 76, 492-509.

Biane, J.S., Takashima, Y., Scanziani, M., Conner, J.M., and Tuszynski, M.H. (2016). Thalamocortical Projections onto Behaviorally Relevant Neurons Exhibit Plasticity during Adult Motor Learning. Neuron 89, 1173-1179.

Bopp, R., Holler-Rickauer, S., Martin, K.A.C., and Schuhknecht, G.F.P. (2017). An Ultrastructural Study of the Thalamic Input to Layer 4 of Primary Motor and Primary Somatosensory Cortex in the Mouse. The journal of neuroscience 37, 2435-2448. Bortone, D.S., Olsen, S.R., and Scanziani, M. (2014). Translaminar Inhibitory Cells Recruited by Layer 6 Corticothalamic Neurons Suppress Visual Cortex. Neuron 82, 474-485.

Bruno, R.M. (2011). Synchrony in sensation. Current opinion in neurobiology 21, 701-708. Bruno, R.M., and Sakmann, B. (2006). Cortex Is Driven by Weak but Synchronously Active Thalamocortical Synapses. Science 312, 1622-1627.

Celnik, P. (2015). Understanding and modulating motor learning with cerebellar stimulation. Cerebellum 14, 171-174.

Churchland, M.M., Cunningham, J.P., Kaufman, M.T., Foster, J.D., Nuyujukian, P., Ryu, S.I., and Shenoy, K.V. (2012). Neural population dynamics during reaching. Nature 487, 51-56. Cortes, C., and Vapnik, V. (1995). Support-vector networks. Machine Learning 20, 273-297. Cruikshank, S.J., Lewis, T.J., and Connors, B.W. (2007). Synaptic basis for intense thalamocortical activation of feedforward inhibitory cells in neocortex. Nature neuroscience $10,462-468$.

Delevich, K., Tucciarone, J., Huang, Z.J., and Li, B. (2015). The Mediodorsal Thalamus Drives Feedforward Inhibition in the Anterior Cingulate Cortex via Parvalbumin Interneurons. journal of Neuroscience 35, 5743-5753.

Estebanez, L., Hoffmann, D., Voigt, B.C., and Poulet, J.F.A. (2017). Parvalbumin-Expressing GABAergic Neurons in primary Motor Cortex Signal Reaching. Cell reports 20, 308-318. Gabernet, L., Jadhav, S.P., Feldman, D.E., Carandini, M., and Scanziani, M. (2005).

Somatosensory Integration Controlled by Dynamic Thalamocortical Feed-Forward Inhibition. Neuron 48, 315-327.

Geiger, J.R.P., Melcher, T., Koh, D.-S., Sakmann, B., Seeburg, P.H., Jonas, P., and Monyer, H. (1995). Relative abundance of subunit mRNAs determines gating and $\mathrm{Ca}+2$ permeability of AMPA receptors in principal neurons and interneurons in rat CNS. Neuron 15, 193-204. 
Nashef et al. 2020

949

950

951

952

953

954

955

956

957

958

959

960

961

962

963

964

965

966

967

968

969

970

971

972

973

974

975

976

977

978

979

980

981

982

983

984

985

986

987

988

989

990

991

992

993

994

995
Haga, T., and Fukai, T. (2019). Extended Temporal Association Memory by Modulations of Inhibitory Circuits. Physical Review Letters 123, 078101.

Hofer, S.B., Ko, H., Pichler, B., Vogelstein, J., Ros, H., Zeng, H., Lein, E., Lesica, N.A., and Mrsic-Flogel, T.D. (2011). Differential connectivity and response dynamics of excitatory and inhibitory neurons in visual cortex. Nature neuroscience 14, 1045.

Holmes, G. (1917). The Symptoms of Acute Cerebellar Injuries due to Gunshot Injuries. 40, 461-535.

Holmes, G. (1939). The Cerebellum of man. Brain : a journal of neurology 62.

Hore, J., and Flament, D. (1988). Changes in motor cortex neural discharge associated with the development of cerebellar limb ataxia. Journal of neurophysiology 60, 1285-1302. Horne, M., and Butler, E. (1995). The role of the cerebello-thalamo-cortical pathway in skilled movement. Prog Neurobiol 46, 199-213.

Hull, C., Isaacson, J.S., and Scanziani, M. (2009). Postsynaptic mechanisms govern the differential excitation of cortical neurons by thalamic inputs. The Journal of neuroscience : the official journal of the Society for Neuroscience 29, 9127-9136.

Isaac, J.T.R., Ashby, M.C., and McBain, C.J. (2007). The Role of the GluR2 Subunit in AMPA Receptor Function and Synaptic Plasticity. Neuron 54, 859-871.

Isomura, Y., Harukuni, R., Takekawa, T., Aizawa, H., and Fukai, T. (2009). Microcircuitry coordination of cortical motor information in self-initiation of voluntary movements. Nature neuroscience $12,1586-1594$.

Ivry, R., and Keele, S. (1989). Timing Functions of the cerebellum. j cogn neurosci 1, 136-152. Izhikevich, E.M. (2003). Simple model of spiking neurons. IEEE Transactions on Neural Networks 14, 1569-1572.

Ji, X.-y., Zingg, B., Mesik, L., Xiao, Z., Zhang, L.I., and Tao, H.W. (2015). Thalamocortical Innervation Pattern in Mouse Auditory and Visual Cortex: Laminar and Cell-Type Specificity. Cerebral Cortex 26, 2612-2625.

Jonas, P., Racca, C., Sakmann, B., Seeburg, P.H., and Monyer, H. (1994). Differences in Ca2+ permeability of AMPA-type glutamate receptor channels in neocortical neurons caused by differential GluR-B subunit expression. Neuron 12, 1281-1289.

Katai, S., Kato, K., Unno, S., Kang, Y., Saruwatari, M., Ishikawa, N., Inoue, M., and Mikami, A. (2010). Classification of extracellularly recorded neurons by their discharge patterns and their correlates with intracellularly identified neuronal types in the frontal cortex of behaving monkeys. The European journal of neuroscience 31, 1322-1338.

Kaufman, M., Churchland, M., Ryu, S., and Shenoy, K. (2014). Cortical activity in the null space: permitting preparation without movement. Nature neuroscience 17, 440-448. Kaufman, M.T., Churchland, M.M., Santhanam, G., Yu, B.M., Afshar, A., Ryu, S.I., and Shenoy, K.V. (2010). Roles of monkey premotor neuron classes in movement preparation and execution. Journal of neurophysiology 104, 799-810.

Kaufman, M.T., Churchland, M.M., and Shenoy, K.V. (2013). The roles of monkey M1 neuron classes in movement preparation and execution. Journal of neurophysiology 110, 817-825. Kerlin, A.M., Andermann, M.L., Berezovskii, V.K., and Reid, R.C. (2010). Broadly Tuned Response Properties of Diverse Inhibitory Neuron Subtypes in Mouse Visual Cortex. Neuron 67, 858-871.

Kloc, M., and Maffei, A. (2014). Target-Specific Properties of Thalamocortical Synapses onto Layer 4 of Mouse Primary Visual Cortex. The journal of neuroscience 34, 15455-15465. Kondo, M., Sumino, R., and Okado, H. (1997). Combinations of AMPA Receptor Subunit Expression in Individual Cortical Neurons Correlate with Expression of Specific Calcium- 
Nashef et al. 2020

996

997

998

999

1000

1001

1002

1003

1004

1005

1006

1007

1008

1009

1010

1011

1012

1013

1014

1015

1016

1017

1018

1019

1020

1021

1022

1023

1024

1025

1026

1027

1028

1029

1030

1031

1032

1033

1034

1035

1036

1037

1038

1039

1040

1041
Binding Proteins. The Journal of neuroscience : the official journal of the Society for Neuroscience 17, 1570-1581.

Kremkow, J., Perrinet, L.U., Masson, G.S., and Aertsen, A. (2010). Functional consequences of correlated excitatory and inhibitory conductances in cortical networks. Journal of computational neuroscience 28, 579-594.

Kumar, S.S., Bacci, A., Kharazia, V., and Huguenard, J.R. (2002). A Developmental Switch of AMPA Receptor Subunits in Neocortical Pyramidal Neurons. journal of Neuroscience 22, 3005-3015.

Luz, Y., and Shamir, M. (2012). Balancing Feed-Forward Excitation and Inhibition via Hebbian Inhibitory Synaptic Plasticity. PLOS Computational Biology 8, e1002334.

McBain, C.J., and Dingledine, R. (1993). Heterogeneity of synaptic glutamate receptors on CA3 stratum radiatum interneurones of rat hippocampus. j Physiol 462, 373-392.

Medina, J., and Lisberger, S.G. (2007). Variation, signal, and noise in cerebellar sensorymotor processing for smooth-pursuit eye movements. Journal of Neuroscience 27, 68326842.

Merchant, H., Naselaris, T., and Georgopoulos, A. (2008). Dynamic sculpting of directional tuning in the primate motor cortex during three-dimensional reaching. The Journal of neuroscience : the official journal of the Society for Neuroscience 28, 9164-9172.

Middleton, F., and Strick, P. (2000). Basal ganglia and cerebellar loops: motor and cognitive circuits. Brain Res Brain Res Rev 31, 236-250.

Middleton, J.W., Omar, C., Doiron, B., and Simons, D.J. (2012). Neural correlation is stimulus modulated by feedforward inhibitory circuitry. The Journal of neuroscience : the official journal of the Society for Neuroscience 32, 506-518.

Mitchell, J.F., Sundberg, K.A., and Reynolds, J.H. (2007). Differential attention-dependent response modulation across cell classes in macaque visual area V4. Neuron 55, 131-141. Mongillo, G., Rumpel, S., and Loewenstein, Y. (2018). Inhibitory connectivity defines the realm of excitatory plasticity. Nature neuroscience 21, 1463-1470.

Nashef, A., Cohen, O., Harel, R., Israel, Z., and Prut, Y. (2019). Reversible Block of Cerebellar Outflow Reveals Cortical Circuitry for Motor Coordination. Cell reports 27, 2608-2619.

Nashef, A., Cohen, O., Israel, Z., Harel, R., and Prut, Y. (2018a). Cerebellar shaping of motor cortical firing is correlated with timing of motor actions. Cell reports 23, 1275-1285.

Nashef, A., Rapp, H., Nawrot, M.P., and Prut, Y. (2018b). Area-specific processing of cerebellar-thalamo-cortical information in primates. Biological Cybernetics 112, 141-152. Onorato, I., Neuenschwander, S., Hoy, J., Lima, B., Rocha, K.-S., Broggini, A.C., Uran, C., Spyropoulos, G., Klon-Lipok, J., Womelsdorf, T., et al. (2019). A Distinct Class of Bursting Neurons with Strong Gamma Synchronization and Stimulus Selectivity in Monkey V1. Neuron.

Peters, A.J., Liu, H., and Komiyama, T. (2017). Learning in the Rodent Motor Cortex. Annual review of neuroscience $40,77-97$.

Plant, K., Pelkey, K.A., Bortolotto, Z.A., Morita, D., Terashima, A., McBain, C.J., Collingridge, G.L., and Isaac, J.T.R. (2006). Transient incorporation of native GluR2-lacking AMPA receptors during hippocampal long-term potentiation. Nature neuroscience 9, 602.

Pouille, F., and Scanziani, M. (2001). Enforcement of temporal fidelity in pyramidal cells by somatic feed-forward inhibition. Science 293, 1159-1163.

Rock, D.M., and Macdonald, R.L. (1995). Polyamine Regulation of N-Methyl-D-Aspartate Receptor Channels. Annual Review of Pharmacology and Toxicology 35, 463-482. 
1042

1043

1044

1045

1046

1047

1048

1049

1050

1051

1052

1053

1054

1055

1056

1057

1058

1059

1060

1061

1062

1063

1064

1065

1066

1067

1068

1069

1070

1071

1072

1073

1074

1075

1076

1077

1078

1079

1080

1081

1082

1083

1084

1085

1086

1087

1088
Ruach, R., Mitelman, R., Sherman, E., Cohen, O., and Prut, Y. (2015). An assumption-free quantification of neural responses to electrical stimulations. J Neurosci Methods.

Sanderson, J.L., Gorski, J.A., and Dell'Acqua, M.L. (2016). NMDA Receptor-Dependent LTD Requires Transient Synaptic Incorporation of Ca2+-Permeable AMPARs Mediated by AKAP150-Anchored PKA and Calcineurin. Neuron 89, 1000-1015.

Schlerf, J.E., Galea, J.M., Bastian, A.J., and Celnik, P.A. (2012). Dynamic modulation of cerebellar excitability for abrupt, but not gradual, visuomotor adaptation. J Neurosci 32, 11610-11617.

Schoonover, C.E., Tapia, J.C., Schilling, V.C., Wimmer, V., Blazeski, R., Zhang, W., Mason, C.A., and Bruno, R.M. (2014). Comparative strength and dendritic organization of thalamocortical and corticocortical synapses onto excitatory layer 4 neurons. J Neurosci 34, 6746-6758.

Shalit, U., Zinger, N., Joshua, M., and Prut, Y. (2012). Descending systems translate transient cortical commands into a sustained muscle activation signal. Cereb Cortex 22, 1904-1914.

Shenoy, K.V., Sahani, M., and Churchland, M.M. (2013). Cortical control of arm movements: a dynamical systems perspective. Annual review of neuroscience 36, 337-359.

Shental, N., Hertz, T., Weinshall, D., and Pavel, M. (2002). Adjustment Learning and Relevant Component Analysis. European Conference on Computer Vision, 181-185.

Soares, D., Goldrick, I., Lemon, R.N., Kraskov, A., Greensmith, L., and Kalmar, B. (2017).

Expression of Kv3.1b potassium channel is widespread in macaque motor cortex pyramidal cells: A histological comparison between rat and macaque. J Comp Neurol 525, 2164-2174. Sohya, K., Kameyama, K., Yanagawa, Y., Obata, K., and Tsumoto, T. (2007). GABAergic Neurons Are Less Selective to Stimulus Orientation than Excitatory Neurons in Layer II/III of Visual Cortex, as Revealed by In Vivo Functional Ca2 Imaging in Transgenic Mice. the journal of neuroscience 27, 2145-2149.

Swadlow, H.A. (2003). Fast-spike interneurons and feedforward inhibition in awake sensory neocortex. Cereb Cortex 13, 25-32.

Tasic, B., Yao, Z., Graybuck, L.T., Smith, K.A., Nguyen, T.N., Bertagnolli, D., Goldy, J., Garren, E., Economo, M.N., Viswanathan, S., et al. (2018). Shared and distinct transcriptomic cell types across neocortical areas. Nature 563, 72-78.

Thiele, A., Delicato, L.S., Roberts, M.J., and Gieselmann, M.A. (2006). A novel electrodepipette design for simultaneous recording of extracellular spikes and iontophoretic drug application in awake behaving monkeys. J Neurosci Methods 158, 207-211.

Trainito, C., von Nicolai, C., Miller, E.K., and Siegel, M. (2019). Extracellular spike waveform dissociates four functionally distinct cell classes in primate cortex. Current Biology.

Ugawa, Y., Day, B.L., Rothwell, J.C., Thompson, P.D., Merton, P.A., and Marsden, C.D. (1991). Modulation of motor cortical excitability by electrical stimulation over the cerebellum in man. J Physiol 441, 57-72.

Ugawa, Y., Uesaka, Y., Terao, Y., Hanajima, R., and Kanazawa, I. (1995). Magnetic stimulation over the cerebellum in humans. Ann Neurol 37, 703-713.

Vogels, T.P., Sprekeler, H., Zenke, F., Clopath, C., and Gerstner, W. (2011). Inhibitory Plasticity Balances Excitation and Inhibition in Sensory Pathways and Memory Networks. Science 334, 1569-1573.

Wang, H.-P., Spencer, D., Fellous, J.-M., and Sejnowski, T.J. (2010). Synchrony of Thalamocortical Inputs Maximizes Cortical Reliability. Science 328, 106-109.

Xue, M., Atallah, B.V., and Scanziani, M. (2014). Equalizing excitation-inhibition ratios across visual cortical neurons. Nature 511, 596-600. 
bioRxiv preprint doi: $\mathrm{https}$ ://doi.org/10.1101/2020.10.20.346775; this version posted October 21, 2020. The copyright holder for this preprint (which was not certified by peer review) is the author/funder, who has granted bioRxiv a license to display the preprint in perpetuity. It is made available under aCC-BY-NC-ND 4.0 International license.

Nashef et al. 2020

1089 Yu, J., Hu, H., Agmon, A., and Svoboda, K. (2019). Recruitment of GABAergic Interneurons in 1090 the Barrel Cortex during Active Tactile Behavior. Neuron.

1091 Zagha, E., Ge, X., and McCormick, D.A. (2015). Competing Neural Ensembles in Motor Cortex 1092 Gate Goal-Directed Motor Output. Neuron 88, 565-577.

1093

1094 